ARTICLE

\title{
Calcium-controlled conformational choreography in the N-terminal half of adseverin
}

Sakesit Chumnarnsilpa ${ }^{1,2,3}$, Robert C. Robinson ${ }^{2,4}$, Jonathan M. Grimes ${ }^{1,5}$ \& Cedric Leyrat $^{1}$

Adseverin is a member of the calcium-regulated gelsolin superfamily of actin-binding proteins. Here we report the crystal structure of the calcium-free $\mathrm{N}$-terminal half of adseverin (iA1-A3) and the $\mathrm{Ca}^{2+}$-bound structure of $\mathrm{A} 3$, which reveal structural similarities and differences with gelsolin. Solution small-angle X-ray scattering combined with ensemble optimization revealed a dynamic $\mathrm{Ca}^{2}+$-dependent equilibrium between inactive, intermediate and active conformations. Increasing calcium concentrations progressively shift this equilibrium from a main population of inactive conformation to the active form. Molecular dynamics simulations of $\mathrm{iA} 1-\mathrm{A} 3$ provided insights into $\mathrm{Ca}^{2}{ }^{+}$-induced destabilization, implicating a critical role for the A2 type II calcium-binding site and the A2A3 linker in the activation process. Finally, mutations that disrupt the $A 1 / A 3$ interface increase $\mathrm{Ca}^{2+}$-independent $\mathrm{F}$-actin severing by $\mathrm{A} 1-\mathrm{A} 3$, albeit at a lower efficiency than observed for gelsolin domains G1-G3. Together, these data address the calcium dependency of A1-A3 activity in relation to the calcium-independent activity of G1-G3.

\footnotetext{
${ }^{1}$ Division of Structural Biology, University of Oxford, Henry Wellcome Building for Genomic Medicine, Oxford OX3 7BN, UK. ${ }^{2}$ Institute of Molecular and Cell Biology, A*STAR (Agency for Science, Technology and Research), Biopolis, Singapore 138673, Singapore. ${ }^{3}$ School of Biochemistry, Institute of Science, Suranaree University of Technology, 111 University Avenue, Muang, Nakhon Ratchasima 30000, Thailand. ${ }^{4}$ Department of Biochemistry, National University of Singapore, 8 Medical Drive, Singapore 117597, Singapore. ${ }^{5}$ Science Division, Diamond Light Source Ltd, Diamond House, Harwell Science and Innovation Campus, Didcot, Oxfordshire OX11 ODE, UK. Correspondence and requests for materials should be addressed to C.L. (email: cedric@strubi.ox.ac.uk), to S.C. (email: sakesit@sut.ac.th) or to J.M.G. (email: jonathan@strubi.ox.ac.uk).
} 
A dseverin (also known as scinderin) is a member of the gelsolin superfamily of $\mathrm{Ca}^{2+}$-dependent actin-binding proteins, which regulate cytoskeletal dynamics by severing, bundling and capping of actin filaments ${ }^{1}$. The gelsolin superfamily groups a number of modular, evolutionary-related proteins that are composed of 3-6 globular gelsolin homology (GH) domains often fused to non-GH N- and/or C-terminal extensions ${ }^{2}$. In humans, family members include gelsolin, adseverin, villin, supervillin, advillin, villin-like protein, flightless I and CapG (reviewed in ref. 3). Adseverin was first identified in chromaffin cells of bovine adrenal medulla, where it acts as an actin depolymerizing agent that disassembles the cortical network of actin filaments in response to bursts of intracellular calcium, leading to exocytosis of secretary vesicles ${ }^{4-6}$. Adseverin has also been shown to be involved in the regulation of osteoclastogenesis ${ }^{7}$ and thrombocytic ${ }^{8}$, chondrocytic ${ }^{9}$ and odontoblastic differentiation ${ }^{10}$, as well as in cisplatin resistance in bladder cancer ${ }^{11}$. Recently, several studies revealed a role for adseverin in the proliferation of lung carcinoma ${ }^{12}$, and gastric ${ }^{13}$ and prostate cancer cell lines ${ }^{14}$.

Adseverin is composed of six GH domains (A1-A6) and shares $60 \%$ overall sequence identity with gelsolin (G1-G6). Adseverin and gelsolin have been shown to have two calcium-binding events, with Kds of 0.6 and $3 \mu \mathrm{M}$ for adseverin ${ }^{4}$, and $\mathrm{Kds}$ of 0.3 and $1.2 \mu \mathrm{M}$ for gelsolin ${ }^{15}$. Low-affinity calcium association occurs with Kds of 25 and $200 \mu \mathrm{M}$ for gelsolin ${ }^{16}$. Crystallographic studies revealed that these binding events involve the association of eight calcium ions, six of which bind at a conserved site on each domain (type II), whereas the remaining two are also stabilized by contacts with actin (type I; refs 17-20). Both proteins are able to sequester two monomers of actin, as well as sever and cap actin filaments through distinct $\mathrm{Ca}^{2+}$-induced domain rearrangements of the two halves of the molecule $e^{4,17,18,21-23}$. In addition, both proteins are inhibited by membrane lipids such as PIP and $\operatorname{PIP}_{2}$, although adseverin is additionally rendered inactive by phosphatidylinositol and phosphatidylserine ${ }^{24,25}$.

The crystal structure of calcium-free gelsolin ${ }^{26}$ revealed an inactive, globular state, where all the actin-binding sites are obscured by three $\mathrm{Ca}^{2+}$-dependent latches. In this state, the two halves of the molecule interact and adopt a pseudo-symmetric conformation in which the major actin-binding sites on domains G1 and G4 are, respectively, buried by interactions with G3 and G6, while the actin-binding site on G2 is masked by interactions with the C-terminal $\alpha$-helical extension, which is absent in adseverin. In this conformation, the long $\alpha$-helices of G3 and G6 adopt a kinked structure, which is straightened by the $\mathrm{Ca}^{2+}$-induced release of the latches ${ }^{27}$.

The structures of the $\mathrm{N}$ - and $\mathrm{C}$-terminal halves of gelsolin in complex with actin ${ }^{17,18}$ have demonstrated that distinct $\mathrm{Ca}^{2+}$-induced domain rearrangements of each half of the molecule are crucial for actin binding. The $\mathrm{N}$-terminal half adopts a conformation in which a new $\mathrm{Ca}^{2+}$-stabilized G2:G3 interface is formed while G1 dissociates from the rest of the molecule to bind in the groove between actin subdomains 1 and 3 (refs 18,20). In contrast, the C-terminal half adopts a compact V-shaped structure where the G4/G6 latch is released by flipping over G6 to create a new G5:G6 interface ${ }^{17,22}$. Small-angle X-ray scattering (SAXS) studies showed a significant calcium-induced increase in the molecular dimensions of gelsolin, and ab initio reconstructions suggested an opening of the two halves and domain rearrangements consistent with the architecture of the active forms observed crystallographically ${ }^{28}$.

Despite extensive homology between adseverin and gelsolin, adseverin lacks the gelsolin C-terminal $\alpha$-helix latch, a feature that likely explains a less complex relationship between severing rate and calcium concentration than observed for gelsolin ${ }^{21}$. In addition, the actin filament severing and capping activities of the $\mathrm{N}$-terminal half of adseverin (A1-A3) are $\mathrm{Ca}^{2}+$ dependent ${ }^{29}$, whereas the same activities of G1-G3 are $\mathrm{Ca}^{2+}$ independent ${ }^{30}$. These differences, combined with similar activated structures and actin sequestering properties of the C-terminal halves, led to the proposal that the activation process of adseverin is dominated by calcium binding to its $\mathrm{N}$-terminal half ${ }^{23}$. Recently, SAXS studies demonstrated that G1-G3 adopts an active conformation in the absence of calcium ${ }^{31}$, suggesting that the $\mathrm{Ca}^{2+}$-independent severing activity of this fragment results from instability of the G1/G3 latch in the absence of interaction with G4-G6 and the C-terminal $\alpha$-helix latch.

Here we investigated the structure, dynamics and calcium activation of A1-A3 by using a combination of X-ray crystallography, SAXS, molecular dynamics simulations (MDS) and ensemble optimization. We crystallized $\mathrm{A} 1-\mathrm{A} 3$ in the presence of EGTA, revealing a compact auto-inhibited structure similar to the conformational state observed in inactive full-length gelsolin. Unexpectedly, attempts to crystallize the active form of A1-A3 in the presence of $5 \mathrm{mM}$ calcium led to a structure of $\mathrm{Ca}^{2+}$-bound $\mathrm{A} 3$ following protein degradation within the crystallization drop. Using SAXS-based atomic ensemble optimization, we were able to draw a comprehensive picture of the conformational landscape of $\mathrm{A} 1-\mathrm{A} 3$ and track $\mathrm{Ca}^{2+}$-induced conformational changes. Classical MDS provided insights into the calcium-induced destabilization of inactive $\mathrm{A} 1-\mathrm{A} 3$, and revealed the location of potential low-affinity calcium-binding sites. Finally, actin depolymerization assays showed that mutations that destabilize the inactive A1:A3 interface increase A1-A3 severing activity in calcium-free conditions, demonstrating the importance of this latch.

\section{Results}

Calcium-free A1-A3 displays an inactive conformation. Purified recombinant A1-A3, supplemented with $5 \mathrm{mM}$ EGTA, crystallized in space group C2 and its structure was solved by molecular replacement using the structure of G1-G3 excised from inactive full-length gelsolin as the search model and refined to a resolution of $2.9 \AA$ (Fig. 1a and Table 1). Each domain adopts the classic GH fold, which is organized around a central five-stranded mixed $\beta$-sheet sandwiched between a long $\alpha$-helix running approximately parallel to the strands, and a shorter $\alpha$-helix running approximately perpendicular to the strands. The domains adopt the compact arrangement characteristic of inactive gelsolin, with A1 sandwiched between A2 and A3. The structure appears to be relatively rigid, with $\mathrm{B}$-factors indicating that flexibility is limited mainly to the extended A2A3 linker and the short $\alpha$-helices of A1 and A2 (Supplementary Fig. 1a). In this inactive conformation, the long $\alpha$-helix of A3 is kinked to enable the formation of the $\mathrm{A} 1 / \mathrm{A} 3$ latch, resulting in the masking of the major actin-binding interface on A1. The inactive forms of A1-A3 (iA1-A3) and G1-G3 (iG1-G3) are highly similar with an overall root mean squared deviation (r.m.s.d.) in $\mathrm{C} \alpha$ positions of $1.19 \AA$ over 337 aligned residues, highlighting the structural conservation between gelsolin and adseverin (Fig. 1b). It is noteworthy that contrary to adseverin, iG1-G3 is unstable in the absence of G4-G6, leading to an open conformation of $\mathrm{G} 1-\mathrm{G} 3$ in the presence of EGTA ${ }^{31}$. The iA1-A3 crystal lattice is stabilized by a number of crystal contacts, including a relatively large packing interface of $\sim 1,518 \AA^{2}$ (Supplementary Fig. 1b). Interestingly, comparisons with full-length gelsolin show that this interface largely buries the surfaces in $\mathrm{AA} 1-\mathrm{A} 3$ that bind the C-terminal half of the molecule, suggesting that the crystal environment partially mimics the inactive full-length protein (Supplementary Fig. 1c). 
a

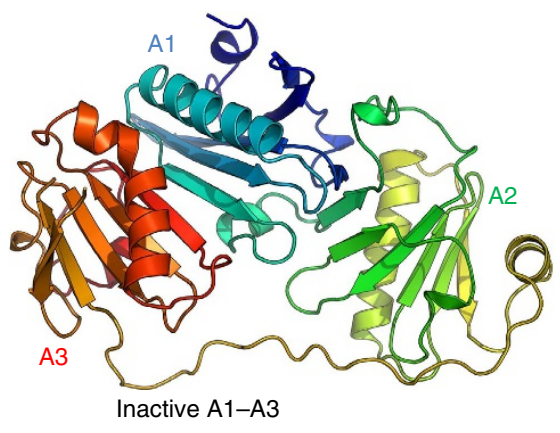

b

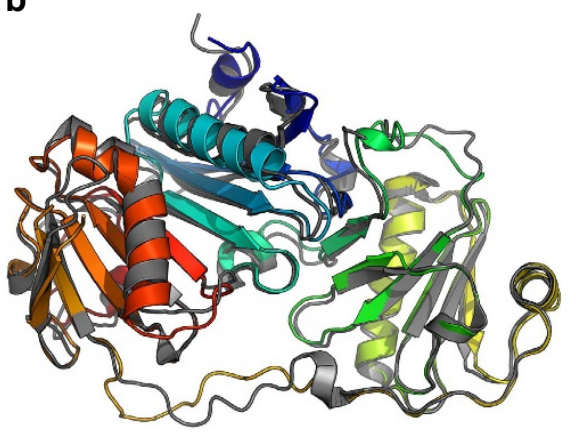

C

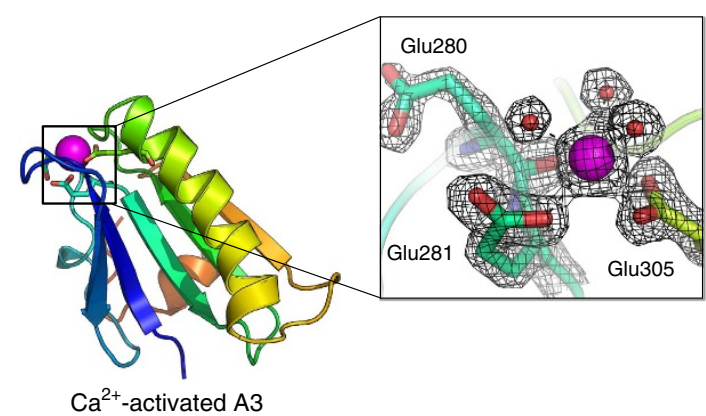

d

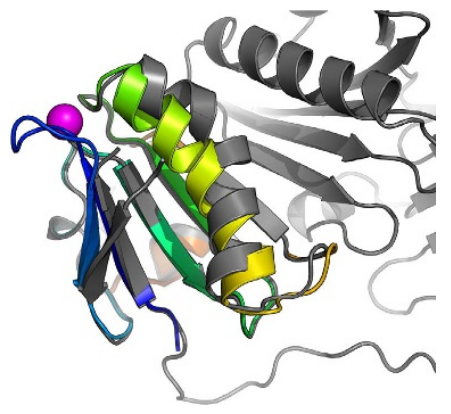

Figure 1 | Crystal structures of the $\mathbf{N}$-terminal half of adseverin in calcium-free conditions (iA1-A3) and of calcium-bound domain $\mathbf{A 3}$ (aA3). (a) The structure of iA1-A3 (residues 6 6349) is shown in cartoon representation and coloured from blue to red ( $\mathrm{N}$ - to $\mathrm{C}$ terminus). (b) Comparison of the structure of iA1-A3 with domains iG1-G3 of gelsolin excised from the structure of full-length gelsolin in calcium-free conditions (PDB 1D0N) ${ }^{26}$, showing a similar compact arrangement of the domains that masks the conserved actin-binding interfaces on A1 and A2. G1-G3 is shown in grey cartoon representation. (c) The calcium-bound structure of adseverin domain A3 (aA3, residues 248-349), shown in cartoon representation and coloured from blue to red ( $\mathrm{N}$ - to $\mathrm{C}$ terminus). The calcium ion is shown as a magenta sphere with coordinating residues as sticks. Inset: close up of the calcium-binding site, showing the coordination of the calcium ion by the Glu280 carbonyl, monodentate Glu281 and bidentate Glu305 side chains, as well as three water molecules. The $2 F_{\mathrm{o}}-F_{\mathrm{c}}$ electron density map contoured at $1.5 \sigma$ is shown as a mesh. (d) Comparison of the structure of aA3 (coloured as blue to red rainbow) and $\mathrm{A} 1-\mathrm{A} 3$ (in grey). Superimposition highlights the conformational changes in the second $\beta$-strand and the long $\alpha$-helix of $A 3$ in response to calcium binding and dissociation from A1.

Calcium-bound structure of A3. Attempts to crystallize the active form of $\mathrm{A} 1-\mathrm{A} 3$ (aA1-A3) using a 5-mM $\mathrm{CaCl}_{2}$ supplemented protein sample led to the appearance of a single crystal after $\sim 80$ days, from which a 1.8- $\AA$ data set was collected. Cell content analysis indicated that the asymmetric unit was too small to accommodate more than a single GH domain, and the structure was solved by molecular replacement using gelsolin domain G3 excised from the structure of actin-bound G1-G3 as a search model (aG3) (PDB 1RGI). This finding was surprising given that homology with gelsolin suggests that in the active form, domains $\mathrm{A} 2$ and $\mathrm{A} 3$ interact through a $\mathrm{Ca}^{2+}$-stabilized interface, while A1 would be separated by the A1A2 linker ${ }^{18,20}$; it would thus have been expected that degradation would lead to a structure of isolated $\mathrm{A} 1$, or a compact, $\mathrm{Ca}^{2+}$-stabilized structure of $\mathrm{A} 2-\mathrm{A} 3$, rather than the $\mathrm{Ca}^{2+}$-bound $\mathrm{A} 3$ structure obtained here.

The conformation of aA3 is highly similar to aG3, with a r.m.s.d. in $\mathrm{C} \alpha$ positions of $0.82 \AA$ over $93 \mathrm{C} \alpha$ atoms, and differs from inactive iA3 and iG3 by the position of the second $\beta$-strand and the straightening of the long $\alpha$-helix (Fig. 1c,d). The structure is stabilized by $\mathrm{Ca}^{2}+$ coordination at the type II binding site, which involves interactions with Glu280 carbonyl, the carboxylates of Glu281 and Glu305, and three water molecules with bond distances ranging between 2.3 and $2.5 \AA$ (Fig. 1c, inset, and Supplementary Fig. 1d). As previously noted for gelsolin, this $\mathrm{Ca}^{2}+$-bound conformation is incompatible with the formation of the A1-A3 latch (Fig. 1d), suggesting that calcium binding at A3 moves A1-A3 away from the inactive conformation through straightening of the A3 long helix resulting in the disruption of the A1:A3 interface ${ }^{18,27}$.

Taken together, these results indicate that the $\mathrm{Ca}^{2+}$-induced conformational changes in adseverin mirror those observed in gelsolin, although in isolation $\mathrm{iA} 1-\mathrm{A} 3$ retains the inactive conformation that is lost by isolated $\mathrm{Ca}^{2+}$-free $\mathrm{G} 1-\mathrm{G} 3$. Furthermore, the structure of aA3 obtained by degradation of $\mathrm{A} 1-\mathrm{A} 3$ in the presence of calcium indicates that A3 may exist in a conformational state where it is distant from A1 and A2.

SAXS experiments. Next, we investigated the structure and calcium activation of A1-A3 using solution SAXS. Scattering profiles for A1-A3 were initially measured in the presence of 2 or $10 \mathrm{mM}$ EGTA, and with increasing concentrations of $\mathrm{CaCl}_{2}$ $\left(10-500 \mu \mathrm{M}\right.$ of free $\left.\mathrm{Ca}^{2+}\right)$, at protein concentrations of $1,2,4$ and $8 \mathrm{mg} \mathrm{ml}^{-1}$ (Fig. 2a, curve series 1-4). The samples were aggregation free, as evidenced by the linearity of the Guinier region (Supplementary Fig. 2), with radii of gyration $\left(R_{\mathrm{g}}\right)$ ranging between 2.6 and $3.2 \mathrm{~nm}$, and molecular weight estimates ranging from 35 to $42 \mathrm{kDa}$ (Table 2), consistent with the theoretical value of $41.5 \mathrm{kDa}$. The $\mathrm{Ca}^{2+}$-induced changes in the scattering profiles were more pronounced at lower protein concentrations, which is to be expected as the Kds for the high-affinity calcium-binding sites are lower than the protein concentrations used in the experiment $\left(K_{\mathrm{d}}=0.6\right.$ and $3 \mu \mathrm{M}$ versus $\left.c=24-192 \mu \mathrm{M}\right)$. An 


\begin{tabular}{|c|c|c|}
\hline & IA1-A3 (PDB ID: 5A1K) & aA3 (PDB ID: 5A1M) \\
\hline \multicolumn{3}{|l|}{ Data collection } \\
\hline Space group & $\mathrm{C} 2$ & 1422 \\
\hline \multicolumn{3}{|l|}{ Cell dimensions } \\
\hline$a, b, c(\AA)$ & $135.4,65.3,105.2$ & $92.5,92.5,86.9$ \\
\hline$\alpha, \beta, \gamma\left(^{\circ}\right)$ & $90,118.3,90$ & $90,90,90$ \\
\hline Resolution $(\AA)$ & $59.6-2.9(3.0-2.9)$ & $35.5-1.8(1.9-1.8)$ \\
\hline$R_{\text {merge, }} \%$ & $15.3(133.0)$ & $10.8(88.9)$ \\
\hline Average $I / I_{0}$ & $10.4(1.5)$ & $21.6(3.5)$ \\
\hline Completeness, \% & $98(95)$ & $100(97)$ \\
\hline Redundancy & $5.5(5.5)$ & $14.2(14.1)$ \\
\hline \multicolumn{3}{|l|}{ Refinement } \\
\hline Resolution $(\AA)$ & $59.6-2.9(3.0-2.9)$ & $35.5-1.8(1.9-1.8)$ \\
\hline No. of reflections & $17,745(2,818)$ & $13,831(2,615)$ \\
\hline$R_{\text {work }} / R_{\text {free }}$ & $24.0(30.2) / 24.8(33.3)$ & $18.2(20.4) / 18.9(22.5)$ \\
\hline \multicolumn{3}{|c|}{ 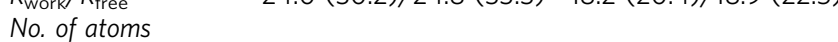 } \\
\hline Protein & 5,402 & 815 \\
\hline Ligand/ion & 0 & 1 \\
\hline Water & 107 & 58 \\
\hline \multicolumn{3}{|l|}{$B$-factors } \\
\hline Protein & 61 & 20 \\
\hline Ligand/ion & 0 & 17 \\
\hline Water & 51 & 28 \\
\hline \multicolumn{3}{|l|}{ r.m.s.d. } \\
\hline Bond lengths $(\AA)$ & 0.008 & 0.008 \\
\hline Bond angles $\left({ }^{\circ}\right)$ & 1.120 & 0.980 \\
\hline
\end{tabular}

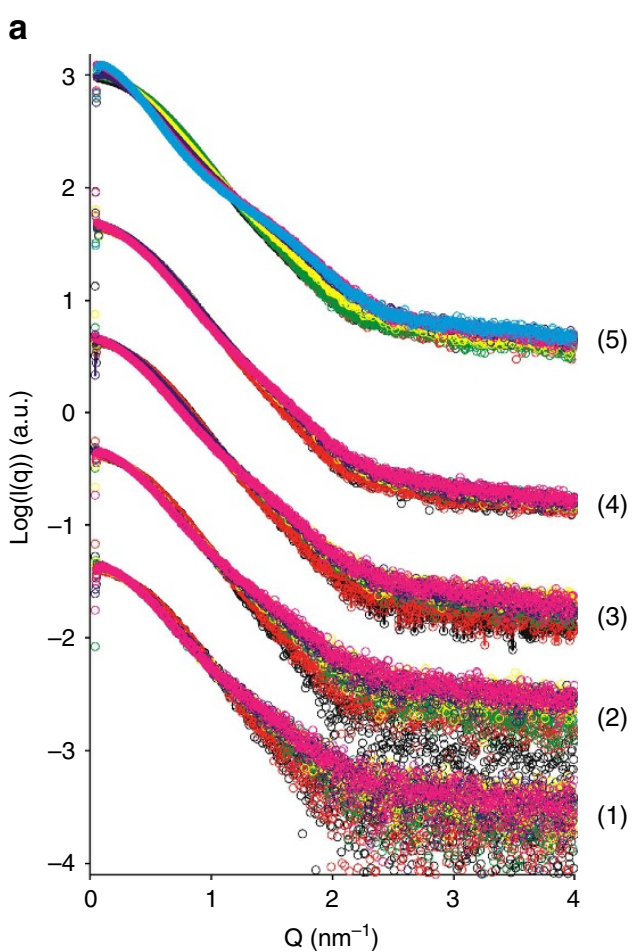

additional calcium titration was performed at $4 \mathrm{mg} \mathrm{ml}^{-1}$ of protein using an increased range of calcium concentrations, in order to check for potential changes at very low (10 or $50 \mathrm{nM})$, as well as higher calcium concentrations ( 2 or $50 \mathrm{mM}$; Fig. 2a, curve series 5, and Table 2). These measurements, performed on a different synchrotron visit using a different protein preparation, showed $\mathrm{Ca}^{2+}$-induced changes that were similar to those observed in the previously acquired data (Fig. 2a, curve series 3, Supplementary Fig. 3a). All measured $R_{\mathrm{g}}$ were, however, smaller by $1-2 \AA$, suggesting mild degradation of the sample (Fig. $2 \mathrm{~b}$ and Table 2). To gain qualitative insights into the $\mathrm{Ca}^{2+}$-induced conformational changes, the data were plotted using the Kratky representation (Fig. 2c). The analysis revealed that A1-A3 is predominantly globular in the absence of calcium, resulting in a bell-shaped curve, and that addition of calcium leads to a more complex profile suggesting modularity and significant interdomain flexibility ${ }^{32}$.

$\mathrm{Ca}^{2+}$-dependent activation of $\mathbf{A 1 - A 3}$. In order to analyse $\mathrm{Ca}^{2+}$-induced changes detected by SAXS, we combined atomistic coarse-grained MDS with ensemble optimization (Supplementary Note 1), demonstrating that A1-A3 transitions from the inactive state (I) to a gelsolin-like active state (A) through two distinct intermediates (Int1 and Int2) characterized by the release of A1/A3 latch and subsequent loss of the A1:A2 interface, respectively (Fig. 3a). All available data could be well fitted using this approach (Fig. 3b), with a goodness of fit $\chi_{\exp }<2$ for $0.08<\mathrm{Q}<5 \mathrm{~nm}^{-1}$ (Table 2), except for data measured in the presence of $50 \mathrm{mM}$ free calcium $\left(\chi_{\exp }=2.05\right)$, which was not analysed further. Data measured at $4 \mathrm{mg} \mathrm{ml}^{-1}$ (Fig. 2a,

b
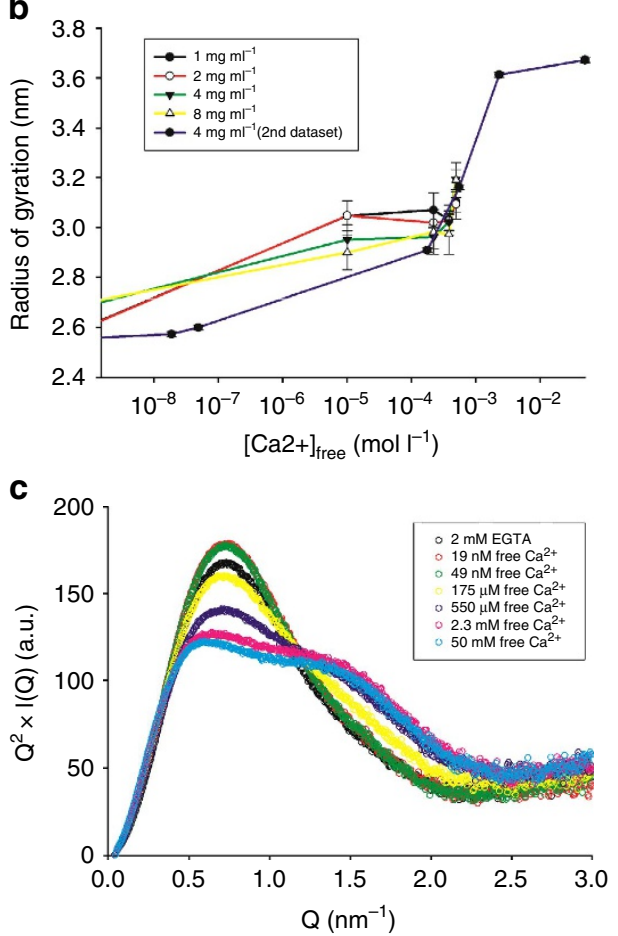

Figure 2 | SAXS experiments. (a) SAXS profiles of A1-A3 in the presence of $10 \mathrm{mM}$ EGTA (black spheres), $2 \mathrm{mM}$ EGTA (red spheres) and with increasing calcium concentrations $\left(10,220,390\right.$ and $490 \mu \mathrm{M} \mathrm{CaCl}_{2}$, green, yellow, purple and pink spheres, respectively) measured at protein concentrations of 1, 2, 4 and $8 \mathrm{mg} \mathrm{ml}^{-1}$ (curve series 1, 2, 3 and 4, respectively). Curve series (5) corresponds to a second calcium titration at $4 \mathrm{mg} \mathrm{ml}^{-1}$ that spans a larger range of calcium concentrations ( $2 \mathrm{mM} \mathrm{EGTA}, 19$ and $49 \mathrm{nM}, 175$ and $550 \mu \mathrm{M}$ and 2.3 and $50 \mathrm{mM}$ free Ca ${ }^{2+}$ ) and was measured on a separate synchrotron visit using a different protein preparation. (b) Changes in radius of gyration as a function of calcium concentration corresponding to the curve series in a: (1) black, (2) red, (3) green, (4) yellow and (5) purple line. (c) Model-free analysis of calcium-induced conformational changes. The data shown in a, curve series (5) is represented as Kratky plots, indicating a transition towards a less globular and more flexible structure. 
Table 2 | SAXS-derived parameters.

\begin{tabular}{|c|c|c|c|c|c|}
\hline $\begin{array}{l}\text { EGTA/free calcium } \\
\text { concentration }\end{array}$ & $c\left(\mathrm{mg} \mathrm{ml}^{-1}\right)$ & MW (kDa) & $R_{\mathrm{g}}(\mathbf{n m})$ & $D_{\max }(n m)$ & $\chi_{\text {exp }}$ \\
\hline 10 mM EGTA & 1.00 & 40 & 2.64 & 9.0 & 0.915 \\
\hline 10 mM EGTA & 2.00 & 42 & 2.62 & 9.1 & 1.123 \\
\hline 10 mM EGTA & 4.00 & 39 & 2.65 & 9.1 & 1.524 \\
\hline 10 mM EGTA & 8.00 & 40 & 2.67 & 9.3 & 1.674 \\
\hline 2 mM EGTA & 1.00 & 39 & 2.61 & 9.1 & 0.832 \\
\hline $2 \mathrm{mM}$ EGTA & 2.00 & 39 & 2.61 & 9.3 & 1.098 \\
\hline $2 \mathrm{mM}$ EGTA & 4.00 & 39 & 2.69 & 9.1 & 1.198 \\
\hline $2 \mathrm{mM}$ EGTA & 8.00 & 40 & 2.70 & 9.2 & 1.461 \\
\hline $10 \mu \mathrm{M} \mathrm{Ca}{ }^{2+}$ & 1.00 & 36 & 3.05 & 10.0 & 0.803 \\
\hline $10 \mu \mathrm{M} \mathrm{Ca}{ }^{2+}$ & 2.00 & 39 & 3.05 & 10.6 & 0.865 \\
\hline $10 \mu \mathrm{M} \mathrm{Ca}{ }^{2+}$ & 4.00 & 38 & 2.95 & 10.3 & 0.939 \\
\hline $10 \mu \mathrm{M} \mathrm{Ca}{ }^{2+}$ & 8.00 & 39 & 2.90 & 10.1 & 1.291 \\
\hline $220 \mu \mathrm{M} \mathrm{Ca}{ }^{2+}$ & 1.00 & 33 & 3.07 & 10.4 & 0.786 \\
\hline $220 \mu \mathrm{M} \mathrm{Ca}{ }^{2+}$ & 2.00 & 37 & 3.02 & 10.4 & 0.862 \\
\hline $220 \mu \mathrm{M} \mathrm{Ca}{ }^{2+}$ & 4.00 & 38 & 2.96 & 10.3 & 0.947 \\
\hline $220 \mu \mathrm{M} \mathrm{Ca}{ }^{2+}$ & 8.00 & 40 & 2.98 & 10.4 & 1.361 \\
\hline $380 \mu \mathrm{M} \mathrm{Ca}{ }^{2+}$ & 1.00 & 36 & 3.03 & 10.5 & 0.827 \\
\hline $380 \mu \mathrm{M} \mathrm{Ca}^{2+}$ & 2.00 & 36 & 3.03 & 11.5 & 0.862 \\
\hline $380 \mu \mathrm{M} \mathrm{Ca}{ }^{2+}$ & 4.00 & 38 & 3.02 & 10.0 & 1.162 \\
\hline $380 \mu \mathrm{M} \mathrm{Ca}{ }^{2+}$ & 8.00 & 40 & 2.97 & 9.9 & 1.281 \\
\hline $490 \mu \mathrm{M} \mathrm{Ca}{ }^{2+}$ & 1.00 & 35 & 3.10 & 10.5 & 0.830 \\
\hline $490 \mu \mathrm{M} \mathrm{Ca}{ }^{2+}$ & 2.00 & 36 & 3.10 & 10.4 & 0.874 \\
\hline $490 \mu \mathrm{M} \mathrm{Ca}^{2+}$ & 4.00 & 39 & 3.19 & 10.2 & 0.914 \\
\hline $490 \mu \mathrm{M} \mathrm{Ca}^{2+}$ & 8.00 & 40 & 3.19 & 10.5 & 1.131 \\
\hline $2 \mathrm{mM}$ EGTA & 4.00 & 38 & 2.56 & 8.8 & $1.386^{\star}$ \\
\hline 19 nM Ca ${ }^{2}+$ & - & 38 & 2.57 & 8.9 & $1.323^{\star}$ \\
\hline $49 \mathrm{nM} \mathrm{Ca}^{2+}$ & - & 38 & 2.60 & 8.7 & $1.343^{\star}$ \\
\hline $175 \mu \mathrm{M} \mathrm{Ca}{ }^{2+}$ & - & 38 & 2.91 & 9.7 & $1.006^{\star}$ \\
\hline $550 \mu \mathrm{M} \mathrm{Ca}{ }^{2+}$ & - & 39 & 3.16 & 10.9 & $0.947^{\star}$ \\
\hline $2.3 \mathrm{mM} \mathrm{Ca}^{2+}$ & - & 43 & 3.61 & 12.3 & $1.262^{\star}$ \\
\hline $50 \mathrm{mM} \mathrm{Ca}^{2+}$ & - & 43 & 3.67 & 13.5 & $2.053^{\star}$ \\
\hline
\end{tabular}

curve series 5), which showed mild signs of degradation, could, however, only be optimally fitted using ensembles in which the last disordered 15 amino acids were truncated (residues 350-365-part of the A3A4 linker; Table 2 and Supplementary Fig. 4d,e). To understand the nature of $\mathrm{Ca}^{2+}$-induced conformational changes, we analysed the $R_{\mathrm{g}}$ distributions (Supplementary Fig. $4 \mathrm{~b}, \mathrm{c}$ ), the relative populations of I, Int1, Int2 and $\mathrm{A}$ (Fig. $3 \mathrm{c}$ ), and the distribution of $\mathrm{A} 1 / \mathrm{A} 2$ and $\mathrm{A} 2 / \mathrm{A} 3$ interdomain distances as a function of free calcium concentration (Fig. 3d-f and Supplementary Fig. 5).

In the presence of EGTA, the $R_{\mathrm{g}}$ distribution displays a major peak at $2.5 \mathrm{~nm}$, and minor populations with $R_{\mathrm{g}}$ values broadly distributed $\sim 4.0-5.5 \mathrm{~nm}$ (Supplementary Fig. 4b,c), which correspond to $85-90 \%$ inactive conformation, $5 \% \mathrm{~A}$ and $9 \%$ Int1, respectively (Fig. 3c,d and Supplementary Fig. 5a). The open conformation Int 2 is highly disfavoured with a population of $0-1 \%$, suggesting that the transition to the active conformation following release of $\mathrm{A} 3$ is a fast process in these conditions. The presence of the minor populations is apparent in the significant improvement of $\chi_{\exp }$ observed when using an optimized ensemble of 20 models $\left(\chi_{\exp }=1.088\right)$ rather than a single best-fitting model of the inactive state to represent the data $\left(\chi_{\exp }=1.919\right.$; Supplementary Fig. 6a). Accordingly, the minimal number of models required for optimal data fitting is constant at around seven models across the different calcium concentrations used, demonstrating that conformational heterogeneity exists regardless of the presence of calcium (Supplementary Fig. 6b).

The addition of $10 \mu \mathrm{M}$ of free calcium stimulated the exchange process (Fig. 3e and Supplementary Fig. 5b), leading to significant changes in the conformational landscape of A1-A3: the proportion of inactive conformers fell to 50\%, whereas Int1 and Int2 rose to $\sim 15 \%$ each, and active conformers increased to nearly $20 \%$. Analysis of A2/A3 interdomain distances reveals a small population of Int1 conformers sampling distances very close to the inactive state as the A1/A3 latch opens up (Fig. 3e and Supplementary Fig. 5b,d), and the overall distribution of A2/A3 distances within the optimized ensemble is markedly different from that of the pool (Supplementary Fig. 5f). It is noteworthy that this significant increase in conformational dynamics and shift to the active conformation happens at a relatively low free calcium-to-protein ratio (0.1:1), which may be due to binding/unbinding of a single calcium ion to A1-A3 occurring much faster than the conformational changes it induces.

At $\sim 500 \mu \mathrm{M}$ of free calcium (calcium-to-protein ratio 5:1), which is enough to saturate the three conserved type II calcium-binding sites, the population of active conformers reaches a peak at $\sim 50 \%$, while the inactive form drops to $20 \%$. Int1 and Int2 represent, respectively, 10 and 20\% (Fig. 3c,f). This shift towards a main population of active conformers is apparent in $R_{\mathrm{g}}$ distributions (Supplementary Fig. $4 \mathrm{~b}, \mathrm{c}$ ), with the appearance of a broad peak centred at $3 \mathrm{~nm}$. Interestingly, the selected active conformers preferentially sample relatively short distances between $\mathrm{A} 1$ and $\mathrm{A} 2$ compared with the pool distribution (similar to the G1-G2 distance in gelsolin-bound actin; Fig. 3f and Supplementary Fig. 5c,d,h), which may reflect a conformational optimization of the A1/A2 linker for actin binding. At even higher free calcium concentrations $(2 \mathrm{mM}$, calcium-to-protein ratio 20:1), the open state (Int2) becomes favoured over the active 


\section{a}
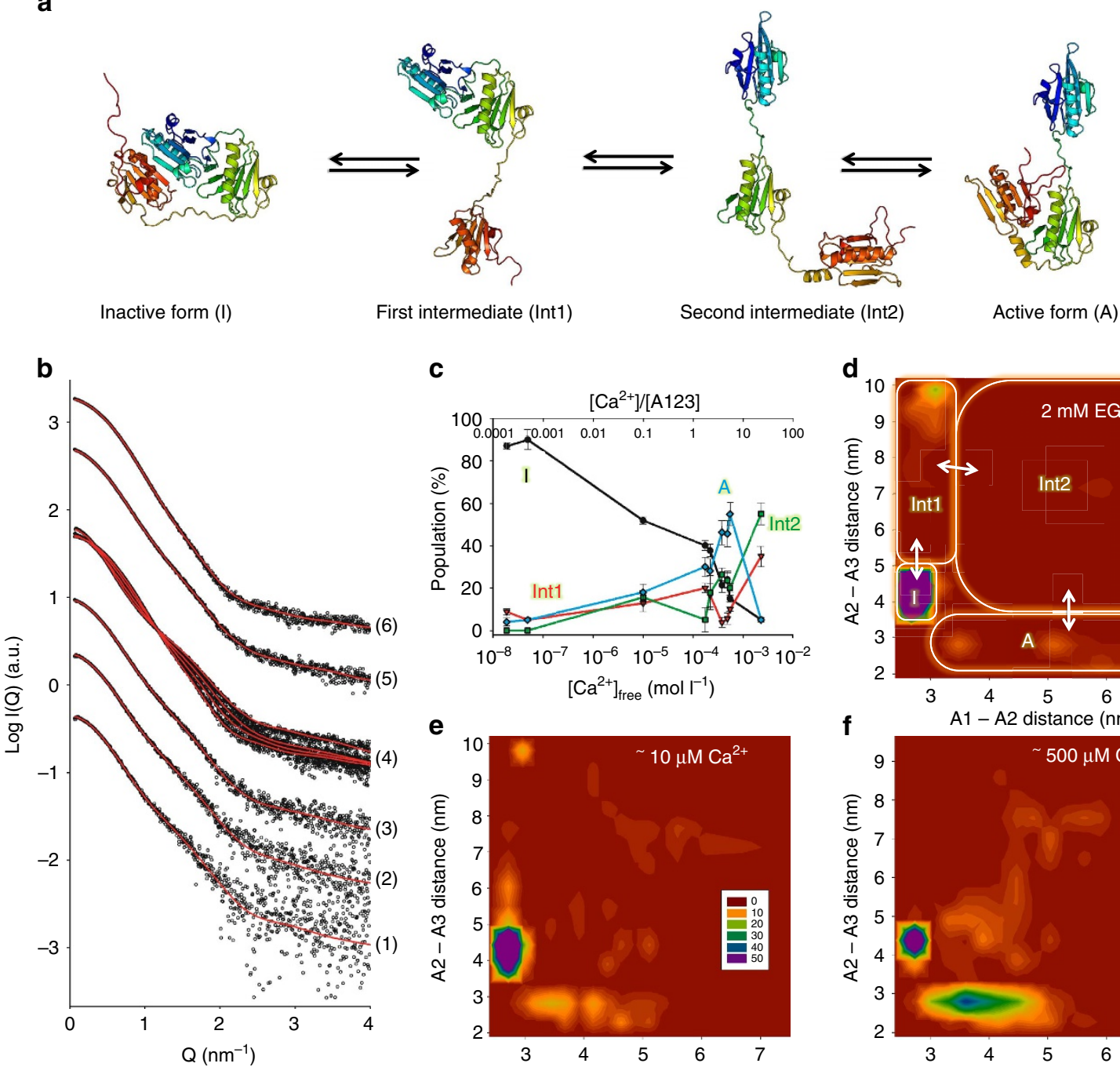

C
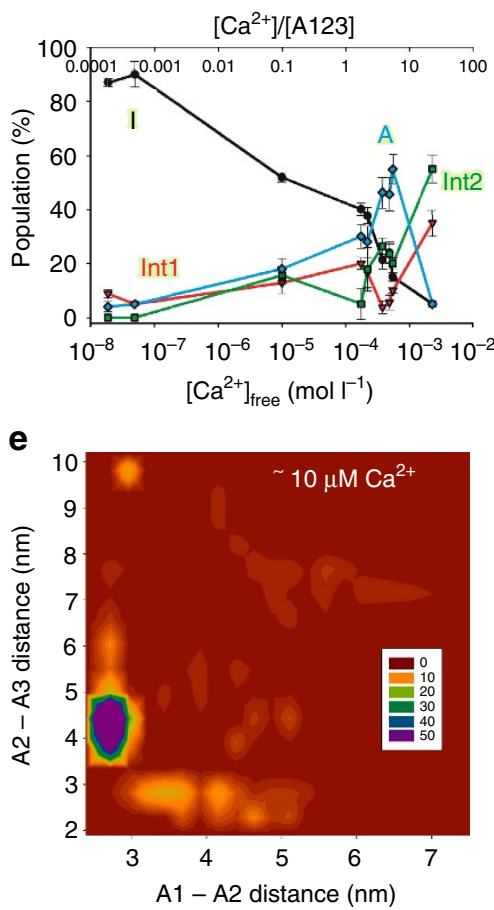

d
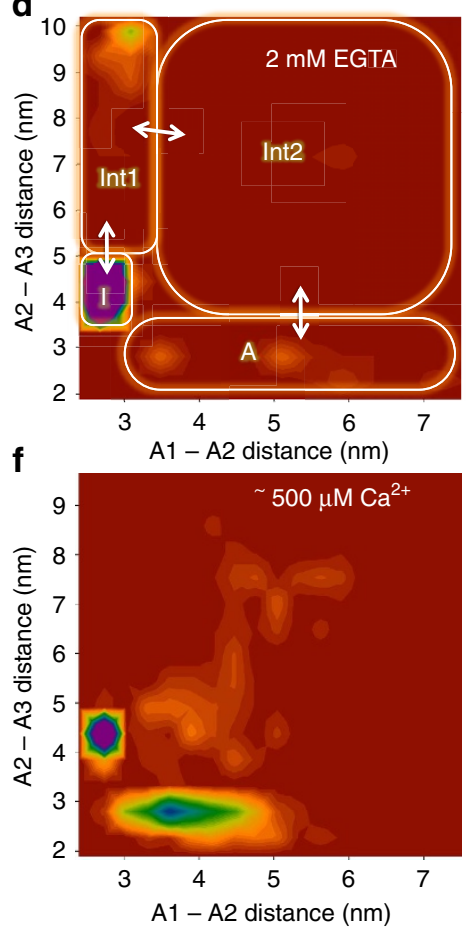

Figure 3 | Proposed mechanism of calcium activation of A1-A3 in solution. (a) Model for A1-A3 transition between the inactive and active (actin-binding competent) states. The release of the A1/A3 latch from the inactive form (I) leads to a first intermediate (Int1), followed by the loss of the A1A2 interface (Int2). Finally, the calcium-stabilized interface between A2 and A3 is formed, resulting in the active conformation (a). (b) Fitted SAXS profiles. For clarity, only a subset of the data shown in Fig. $2 a$ is represented. Experimental data are shown as black spheres and theoretical SAXS profiles for optimized ensembles of 50 models are drawn as red lines. (1) $490 \mu \mathrm{M} \mathrm{CaCl}_{2}, 1 \mathrm{mg} \mathrm{ml}^{-1}$ of A1-A3; (2) $490 \mu \mathrm{M} \mathrm{CaCl}_{2}, 2 \mathrm{mg} \mathrm{ml}^{-1}$ of A1-A3; (3) $490 \mu \mathrm{M} \mathrm{CaCl}$, $4 \mathrm{mg} \mathrm{ml}^{-1}$ of $\mathrm{A} 1-\mathrm{A} 3$; (4) $49 \mathrm{nM}, 175$ and $550 \mu \mathrm{M}$, and $2.3 \mathrm{mM} \mathrm{CaCl}_{2}, 4 \mathrm{mg} \mathrm{ml}^{-1}$ of $\mathrm{A} 1-\mathrm{A} 3$; (5) $490 \mu \mathrm{M} \mathrm{CaCl}_{2}, 8 \mathrm{mg} \mathrm{ml}^{-1}$ of $\mathrm{A} 1-\mathrm{A} 3$; (6) $2 \mathrm{mM}^{-}$ EGTA, $8 \mathrm{mg} \mathrm{ml}^{-1}$ of $A 1-A 3 \mathrm{mg} \mathrm{ml}^{-1}$. (c) Relative populations of inactive (I, black), intermediate (Int1, red and Int2, green) and active (A, blue) conformations of $A 1-A 3$, plotted as a function of free calcium concentration ( $x$ axis, bottom) and ratio of calcium ion over protein ( $x$ axis, top). All data measured at $4 \mathrm{mg} \mathrm{ml}^{-1}$ were used (Fig. 2 A, curve series 3 and 5). Error bars represent the s.d. of ensemble optimization results obtained from 10 independent ensembles of 6,000 models (see Methods section). (d-f) Two-dimensional (2D) histogram representations of the distribution of A1-A2 and A2-A3 interdomain distances (taken between their centres of mass) within the optimized ensembles, using data measured in the presence of $2 \mathrm{mM}$ EGTA (d), $10 \mu \mathrm{M}(\mathbf{e})$ and $550 \mu \mathrm{M}$ free $\mathrm{Ca}^{2+}$ (f). The $2 \mathrm{D}$ histogram was calculated using a binning of $15 \times 15$ and coloured from red to blue (0-50 models).

conformation ( 55 versus $5 \%$ ), while the inactive conformation is reduced to $5 \%$ (Fig. $3 \mathrm{c}$ and Supplementary Fig. 4c). These observations are in line with the increase in the measured $R_{\mathrm{g}}$ $(3.6 \mathrm{~nm}$; Table 2$)$, and the predominance of the open state may explain the degradation of A1-A3 that led to crystallization of A3 in the presence of $5 \mathrm{mM}$ calcium.

MDS of calcium binding to inactive A1-A3. Ensemble optimization demonstrated a complex calcium-induced remodelling of A1-A3 conformational landscape. To gain insights into the initial events that shift the equilibrium towards the active state, we performed explicit solvent MDS of $\mathrm{iA} 1-\mathrm{A} 3$ in the presence of $50 \mathrm{mM} \mathrm{CaCl}_{2}$ or $50 \mathrm{mM} \mathrm{NaCl}$ (Fig. 4). Although well above the physiological range, such high calcium concentrations enable the detection of regions that display conformational sensitivity to calcium on fast timescales, as well as potential low-affinity sites, within reasonable simulation times (for example, ref. 33). For each system, we ran two independent simulations of $1 \mu \mathrm{s}$ and $0.1 \mu \mathrm{s}$ in the presence and absence of calcium, respectively. The protein structure was stable over the course of the simulations, with the r.m.s.d. from the starting structure stabilizing $\sim 3-4 \AA$ after an initial rise during the first $20 \mathrm{~ns}$ (Fig. 4a,b). Analysis of the root mean square fluctuations 

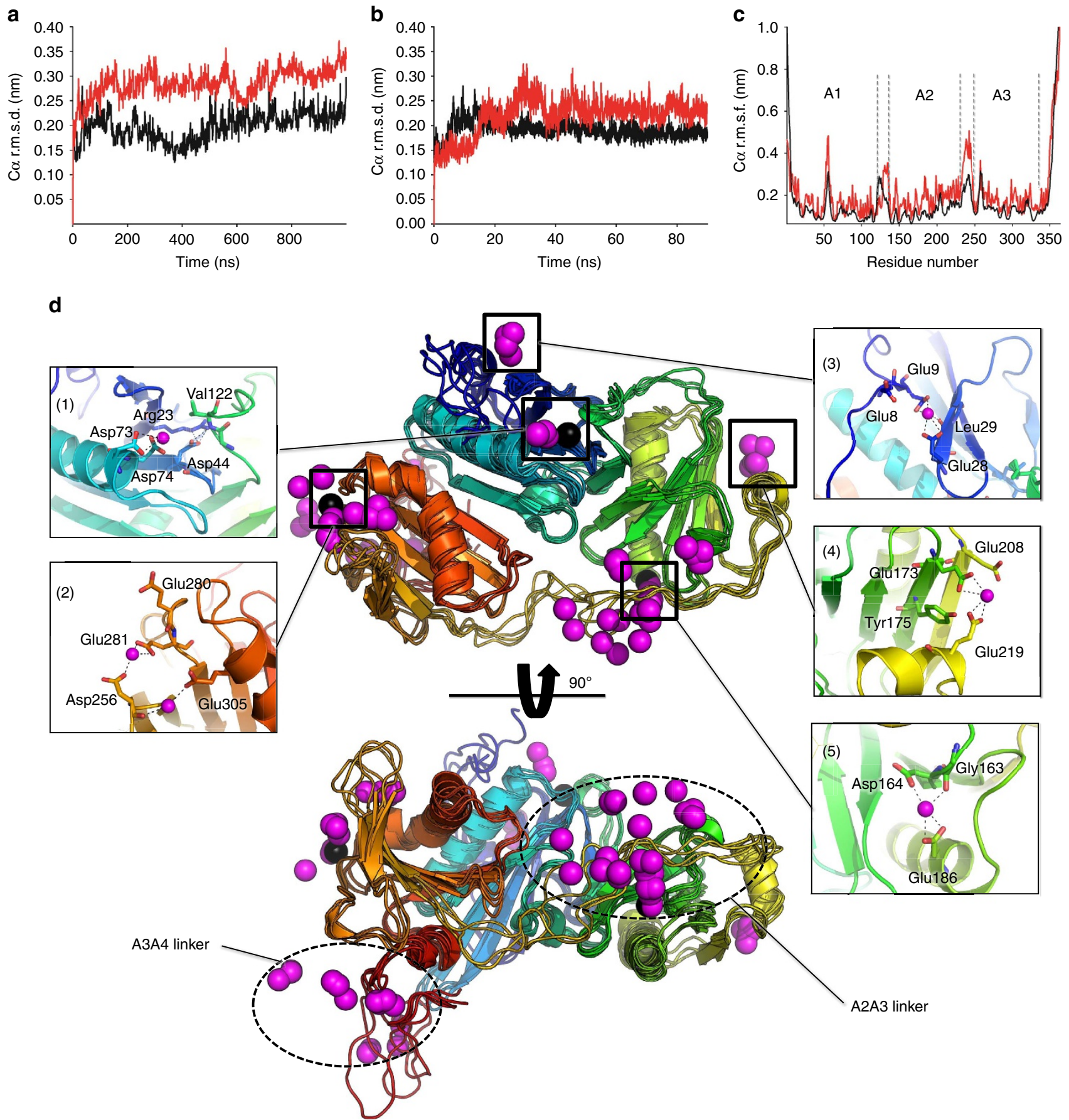

Figure 4 | MDS of A1-A3 in the inactive state. (a,b) r.m.s.d. of $\mathbf{C} \alpha$ atoms from the starting structure in the presence of $50 \mathrm{mM} \mathrm{CaCl} 2(\mathbf{a})$ or $\mathrm{NaCl}(\mathbf{b})$. The results from two independent simulations are shown as black and red lines, respectively. (c) Root mean square fluctuations (r.m.s.f.) of $\mathrm{C} \alpha$ atoms along the protein sequence. The averages of the two independent simulations are shown as black and red lines for systems simulated in the absence and presence of calcium, respectively. (d) Analysis of calcium binding to inactive iA1-A3 by MDS. Calcium ions displaying the lowest time-averaged distance to protein atoms were extracted from eight snapshots taken at $100 \mathrm{~ns}$ of interval and are represented as magenta spheres. Four overlaid snapshots of the protein are shown in two views rotated by $90^{\circ}$ in cartoon representation with the protein backbone coloured from blue to red ( $\mathrm{N}$ - to $\mathrm{C}$ terminus). The protein backbone from four additional snapshots, for which the calcium ions are shown, have been hidden for clarity. Close ups of the calcium-binding sites of interest are shown as insets.

indicated flexibility mainly in the linker regions, which was enhanced by calcium (Fig. 4c).

We identified bound calcium by analysing time-averaged distances between $\mathrm{Ca}^{2+}$ and protein atoms (Fig. 4d). $\mathrm{Ca}^{2+}$ clusters mainly around the conserved type II binding sites (Fig. 4d, inset 1,2 and 5), as well as the A2A3 linker and the $\mathrm{C}$-terminal disordered extension belonging to the A3A4 linker, in a manner that is consistent with the distribution of negative charges on the protein surface (Supplementary Fig. 7). Additional $\mathrm{Ca}^{2}+$ is observed between the short $\alpha$-helices of $\mathrm{A} 1$ and $\mathrm{A} 2$, and their second and fourth $\beta$-strands, respectively (Fig. $4 \mathrm{~d}$, inset 3 and 4). These sites involve calcium coordination by Glu9 and Glu28 carboxylates, and the backbone oxygen of Leu29 in A1, and Glu219 and Glu173 carboxylates and the hydroxyl of Tyr175 in A2. These interactions create additional contacts between the short $\alpha$-helices and $\beta$-strands of $\mathrm{A} 1$ and $\mathrm{A} 2$, and 
may represent low-affinity-binding sites that stabilize the $\mathrm{GH}$ domains at high calcium concentrations.

Examination of $\mathrm{Ca}^{2+}$ dynamics at the high-affinity sites indicates that only the type II binding site at $\mathrm{A} 2$ can bind $\mathrm{Ca}^{2+}$ in the inactive conformation (Fig. 4d, inset 5), showing stable coordination of $\mathrm{Ca}^{2+}$ by Asp164 and Glu186 side chains, as well as Gly163 carbonyl oxygen. In contrast, binding at A1 is hindered by the interaction of Asp44 with Arg23 side chain and Val122 backbone nitrogen, resulting from the interaction with A2 (Fig. 4d, inset 1). Similarly, formation of the type II binding site in A3 is prevented by the kink of the long $\alpha$-helix that interacts with A2, resulting in distances between Glu305, Asp256 and Glu281 that are too large to coordinate calcium tightly (Fig. 4d, inset 2).

Next, we analysed the effect of calcium on the dynamics of the A2A3 linker, which is located close to the type II binding site at A2 (Fig. 5), and in actin-bound G1-G3 contacts calcium through Asp259 side chain (Asp238 in adseverin), thus providing additional stability to the active state ${ }^{20}$. In the absence of calcium, the extended A2A3 linker samples the conformational space in the vicinity of the crystal structure (Fig. 5a). In contrast, calcium perturbs the linker dynamics, leading to an initial increase in flexibility within the first 100-ns of simulation (Fig. 5b) followed by sampling of calcium-induced, non-native conformational states of the linker associated with calcium binding to the type II binding site in A2 and to the acidic patch of the A2A3 linker (Fig. 5a,d). The observation of calcium-induced changes in A2A3 linker dynamics suggests that this linker acts as a calcium-sensitive conformational switch that modulates the stability of the inactive, intermediate and active states.

Impact of A1/A3 interface mutations on F-actin severing. The detailed picture of the $\mathrm{Ca}^{2+}$-dependent activation mechanism of A1-A3 brought by the combination of X-ray crystallography, SAXS and MDS explains its reported $\mathrm{Ca}^{2+}$-dependent severing activity, which contrasts with the open conformation and $\mathrm{Ca}^{2+}$-independent severing of actin filaments described for the equivalent fragment of gelsolin (G1-G3; refs 30,31). These differences prompted us to investigate the contribution of the stability of the A1/A3 latch to severing activity. To this end, two mutants of A1-A3 (Met310Asp and Glu314Ser) were designed that were aimed at disrupting hydrophobic interactions between Met310 and Phe64, and a salt bridge involving Glu314 and Arg97 at the A1:A3 interface (Fig. 6a). Interestingly, structural comparisons show that in gelsolin, the equivalent residue to Glu314 is a serine, resulting in loss of the salt bridge, while the Met310/Phe64 pair is replaced by Leu332/Tyr87, suggesting a potentially less stable G1:G3 interface (Fig. 6b).

F-actin depolymerization by A1-A3 and its mutants, as well as by G1-G3 was monitored by the loss of fluorescence of pyrene-labelled F-actin in the presence of $1 \mathrm{mM}$ EGTA (Fig. 6c) or $500 \mu \mathrm{M} \mathrm{CaCl}_{2}$ (Fig. 6d). In the presence of $\mathrm{Ca}^{2+}$, all proteins showed a similar actin filament depolymerization pattern, with rapid loss of fluorescence. In contrast, in the presence of EGTA, $\mathrm{A} 1-\mathrm{A} 3$ and the M310D and E314S mutants showed reduced severing activity, while only G1-G3 severing activity remained robust, as expected from previous reports ${ }^{29,30}$. Interestingly, A1-A3 severing of $\mathrm{F}$-actin was roughly 10 times slower than in the presence of $\mathrm{Ca}^{2+}$ (Fig. 6c,d). Although the M310D and E314S mutants showed increased activity compared with wild-type A1-A3, they remained much less active than $\mathrm{G} 1-\mathrm{G} 3$ in $\mathrm{Ca}^{2+}$-free conditions, suggesting that the destabilization of the A1/A3 latch in the mutants may only partially shift the equilibrium towards the active state. In addition, M310D was slightly more active than E314S, indicating a larger contribution to destabilizing the interface, as may be expected based on the nature of the mutation (hydrophobic to charged versus charged to hydrophilic).

\section{Discussion}

The structural data presented here have revealed that isolated A1-A3 can adopt, in the crystal and in solution, an inactive conformation (I) characterized by the formation of the A1/A3 latch as observed in calcium-free full-length gelsolin. Using SAXS and ensemble optimization, we have demonstrated that the conformational landscape of $\mathrm{A} 1-\mathrm{A} 3$ is composed of a a

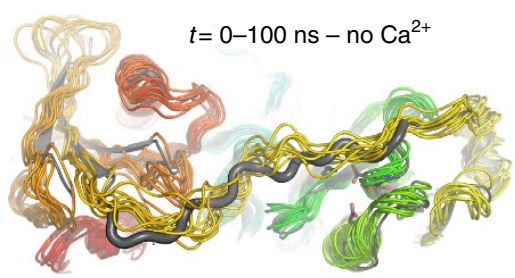

b

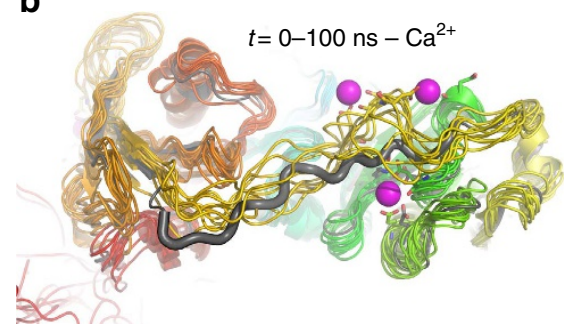

d

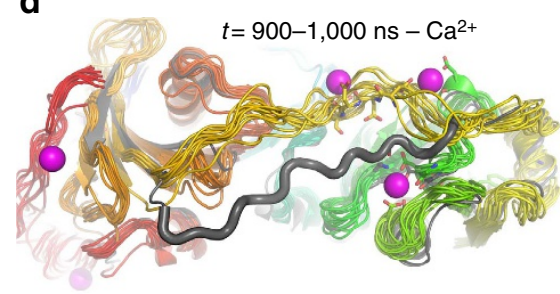

Figure 5 | Effect of calcium on A2A3 linker dynamics as observed in MDS. In each panel, the crystal structure of iA1-A3 is shown in grey cartoons for comparison with the A2A3 linker represented using a larger cartoon loop radius. (a) Linker dynamics in the absence of calcium. Ten overlaid snapshots of the protein taken at $10 \mathrm{~ns}$ of interval (10-100 ns) are shown in cartoon representation with the protein backbone coloured from blue to red ( $\mathrm{N}$ - to $\mathrm{C}$ terminus). (b-d) Linker dynamics in the presence of calcium, illustrating the increased flexibility and deviation from the crystal structure. Ten overlaid snapshots of the protein taken at $10 \mathrm{~ns}$ of interval were extracted from one of the two $1 \mu \mathrm{s}$ MDS of iA1-A3 in the presence of calcium and are shown as rainbow-coloured cartoons (0-90, 400-490 and 900-990 ns in b-d respectively). In each case, bound calcium ions are represented in magenta spheres for one of the snapshots, with coordinating side chains as sticks ( $100 \mathrm{~ns}$ in $\mathbf{b}, 500 \mathrm{~ns}$ in $\mathbf{c}$ and $1,000 \mathrm{~ns}$ in $\mathbf{d}$ ). 
a

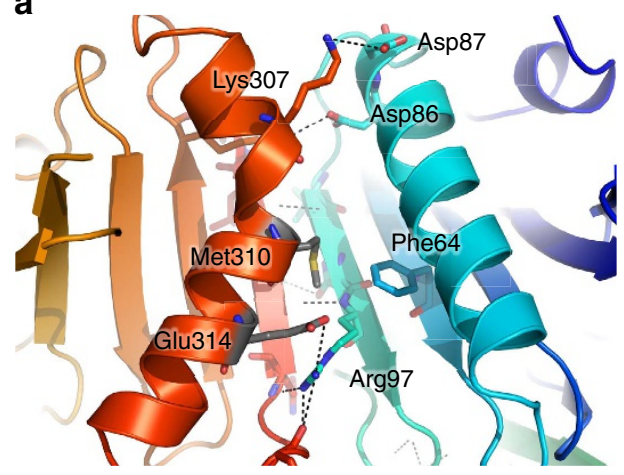

C

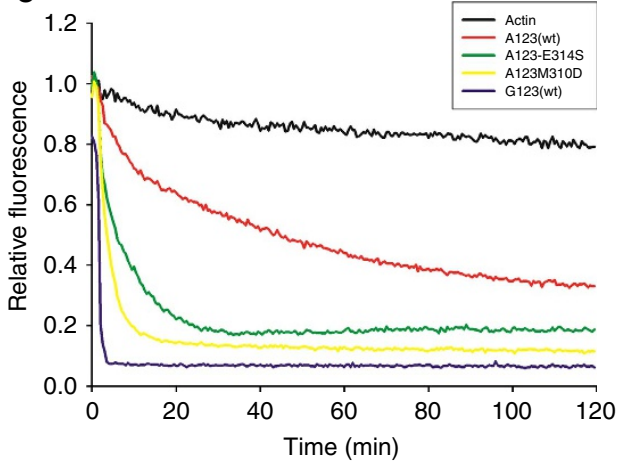

b

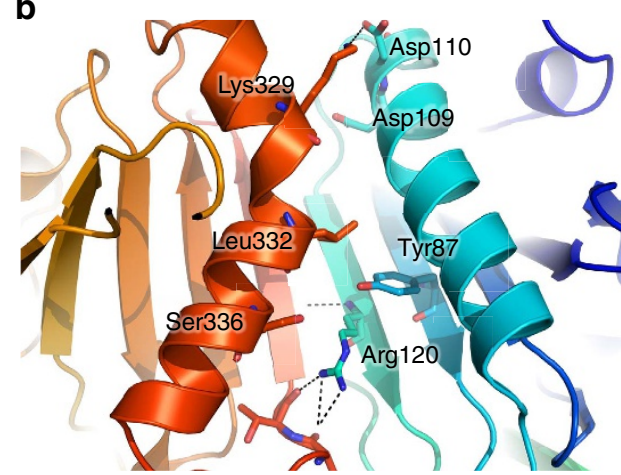

d

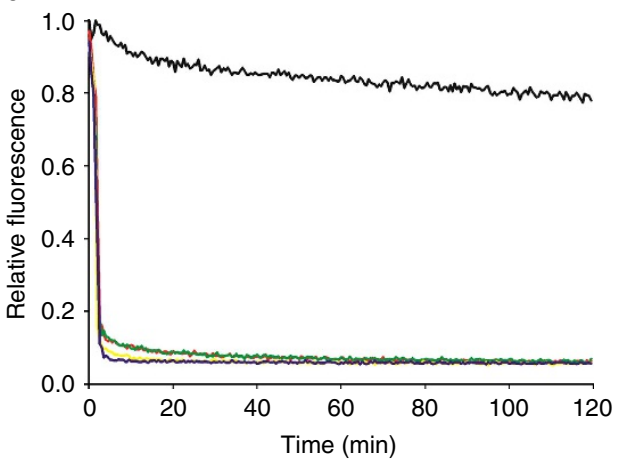

Figure 6 | Calcium-independent actin-severing activity of A1-A3 is increased by mutations that disrupt the A1/A3 interface. (a) Details of the A1/A3 interface. The mutated residues Met310 and Glu314 are shown as grey sticks. (b) Similar view of the G1/G3 interface. (c,d) Actin depolymerization assays. A total of $6 \mu \mathrm{M}$ of each protein (actin control, black; A1-A3wt, red; E314S mutant, green; M310D mutant, yellow; and G1-G3, purple) was added to $6 \mu \mathrm{M}$ of F-actin in the presence of $1 \mathrm{mM} \mathrm{EGTA} \mathrm{(c)} \mathrm{or} 0.5 \mathrm{mM} \mathrm{CaCl}_{2}$ buffer (d).

heterogeneous mixture of dynamically exchanging conformers, where the inactive conformation coexists with activation intermediates and gelsolin-like active conformers in a 9:1 ratio in the absence of calcium. The addition of moderate $(10 \mu \mathrm{M})$-to-high $(500 \mu \mathrm{M})$ calcium concentrations increased conformational exchange, resulting in a larger proportion of intermediate forms (30\%), and progressively shifted the equilibrium towards the active form, up to a maximum of $\sim 55 \%$. These calcium concentrations correlate well with the $\sim 50 \mu \mathrm{M}$ peaks in subplasmalemmal calcium observed in adrenal chromaffin cells ${ }^{34}$, which enable adseverin to depolymerize the cortical network of actin filaments. Interestingly, we found that higher calcium concentrations $(2 \mathrm{mM}$, close to blood plasma calcium concentration of $1.0-1.5 \mathrm{mM}$ ) induced further expansion leading to a main population of open conformers (Int2). Because of its intrinsic disorder, this open conformation may result in faster actin binding and severing through a fly casting mechanism ${ }^{35,36}$, and increase the activity of the protein when present in the extracellular environment, for example, following injury as adseverin is highly expressed in soft tissue ${ }^{3,37}$. The predominance of Int 2 at high calcium concentration may explain the slow degradation of A1-A3 and crystallization of $\mathrm{A} 3$ in the presence of $5 \mathrm{mM}$ calcium owing to the increased accessibility of the A1A2 and A2A3 linkers to proteolytic cleavage. This crystal structure revealed the presence of a single calcium ion bound at the type II binding site, associated with the straightened long $\alpha$-helix that is characteristic of the active forms of gelsolin domains G3 and G6, highlighting the similarity of $\mathrm{Ca}^{2+}$-induced conformational changes in both proteins.

Classical MDS provided detailed insights into the binding of calcium to inactive $\mathrm{A} 1-\mathrm{A} 3$, indicating that binding at $\mathrm{A} 2$ can occur in the inactive state while binding at the other type II calcium sites on A1 and A3 is hindered by interdomain contacts
(Fig. 4). This bound calcium likely plays a major role in shifting the equilibrium towards the active state at low calcium concentrations by perturbing the conformation of the A2A3 linker (Fig. 5), which undergoes significant refolding during the inactive-to-active transition (Fig. 7a). However, an additional, perhaps more important, mechanism by which calcium binding at A2 may shift the conformational landscape is through stabilization of the active A2:A3 interface (Fig. 7b), likely resulting in an increased lifetime of the active conformation. The inaccessibility of the A1 and A3 type II calcium-binding sites in the inactive conformation implies that the increase in the population of active conformers observed at calcium-to-protein ratios $>1$ is a consequence of calcium binding at A1 and A3 in the Int 1 , Int 2 and A conformers, which, in combination with the straightening of the long $\alpha$-helix of A3, prevents the molecule from transitioning back to the inactive form. Finally, we show that very high calcium concentrations destabilize the active A2:A3 interface, leading to a majority of open conformers. Based on the MDS results, we propose that additional calcium binding in the A2A3 linker region, and in particular at the highly acidic stretch 232-DGGDDDD-238, may cause the observed destabilization. Interestingly, potential additional low-affinity sites that might play a role in stabilizing the $\mathrm{A} 1$ and $\mathrm{A} 2$ domain structure were also identified in MDS (Fig. 4), although they have not been verified experimentally. The A2A3 linker appears to be optimized for calcium-induced conformational change through allosteric regulation by association with calcium (Fig. 5). Furthermore, ${ }^{232}$-DGGDDDD- ${ }^{238}$ in the A2A3 linker may offer sequential aspartic acids to the occupied type II calcium-binding site on A2. Thus, as A3 approaches A2, a pathway of calcium/ aspartic acid interactions may lead to the docking of the A2:A3 interface, which is stabilized by the final aspartic acid in the 
a

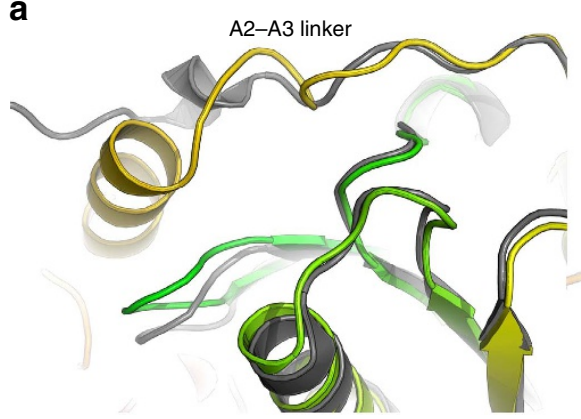

b

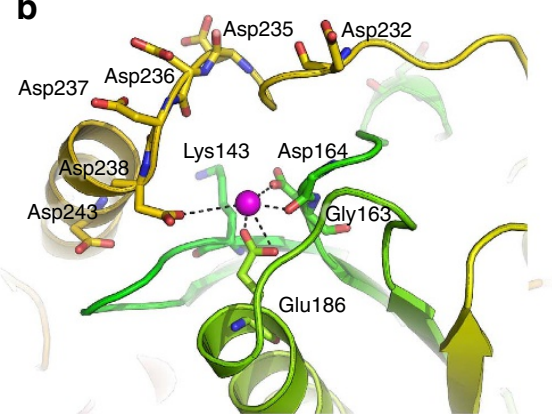

C

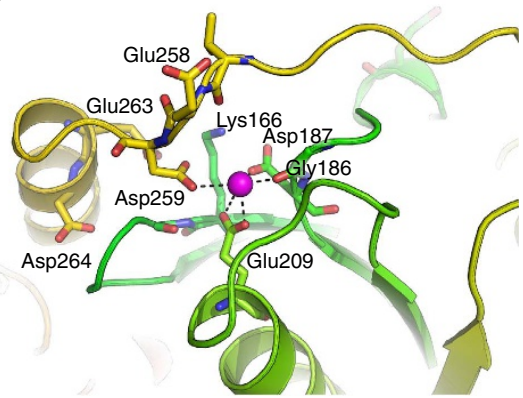

Figure 7 | Role of the A2A3 linker in activation and comparison with gelsolin. (a) Conformation of the A2A3 linker in the inactive structure (grey cartoons) and active model (rainbow-coloured cartoons) of A1-A3. The crystal structure of iA1-A3 was superimposed onto a homology model of the gelsolin-like active conformation through structural alignment of A2. (b,c) Calcium coordination at the type II binding site of domain 2 in the active A1-A3 model (b) and in the crystal structure of actin-bound G1-G3 (PDB 3FFK) (c), highlighting sequence divergence in this region with a more acidic linker in adseverin.

sequence (Asp238) coordinating the type II calcium bound to A2 (Fig. 7). Such functionality in linker regions is beginning to be recognized across a range of multidomain proteins ${ }^{38}$.

Actin depolymerization assays have shown that $\mathrm{A} 1-\mathrm{A} 3$ is able to slowly sever actin filaments in EGTA conditions, although this activity was roughly $10 \%$ of the severing rate observed in the presence of $500 \mu \mathrm{M}$ calcium. Interestingly, this correlates well with the proportion of active conformers detected by SAXS in similar conditions, which are, respectively, 5 and $50 \%$ in the presence of $2 \mathrm{mM}$ EGTA and $500 \mu \mathrm{M}$ calcium. This suggests that $\mathrm{F}$-actin binding by $\mathrm{A} 1-\mathrm{A} 3$ is dominated by conformational selection of the active form, and that the inactive-to-active transition may be relatively slow compared to F-actin binding and severing, preventing population shift from occurring. Alternatively, the large concentration of G-actin present in the assay might hinder population shift through sequestering of G-actin by A1-A3 following an initial binding event to the inactive conformation.

The M310D and E314S mutants of A1-A3 showed increased severing activity in the absence of calcium when compared with wild-type A1-A3, most probably due to a partial shift towards the active conformation, which highlighted the importance of the A1/A3 contacts in stabilizing the inactive state. However, the activity of the mutants in calcium-free conditions remained much lower than for wild-type A1-A3 in the presence of calcium, or G1-G3 that displays fast depolymerization activity regardless of the presence of calcium ${ }^{30}$. The fact that these mutations were unable to completely turn $\mathrm{A} 1-\mathrm{A} 3$ into a $\mathrm{Ca}^{2+}$-independent severer suggests that the high level of activity of G1-G3 does not primarily result from the instability of the inactive state G1-G3. In support of this, comparison of predicted $\Delta^{i} G$ for the interfaces between domains $1-2$ and $1-3$ in iA1-A3 and iG1-G3 using the Pisa Web server ${ }^{39}$ revealed no major difference in stability, and a $\sim 150$-ns MDS of iG1-G3 suggested that the G1:G3 interface is stable on such timescales (Supplementary Fig. 8), also indicating that the domain rearrangements required for activation are slow.

A second structural difference between the two proteins may reside in the stability of the active form, which is tightly influenced by the sequence of the A2A3 linker. Indeed, the concentration of negative charges in the highly acidic stretch ${ }^{232}$-DGGDDDD- ${ }^{238}$ of A1-A3, as well as the loss of ionic interactions between Lys166 and Glu263, may lead to an active structure that is less stable than aG1-G3 (Fig. 7b,c). Consistent with this, SAXS studies of G1-G3 and fragments G1-G2 and G2-G3 showed that G1-G3 mainly adopts an active/open conformation in solution regardless of calcium concentration, explaining its calcium-independent activity ${ }^{31}$. Although ensemble analysis was not performed on this data, the construct used (gelsolin residues 1-371) has the same length as A1-A3 (residues $1-365+5$ residues remaining from the cleaved purification tag), which enables direct comparisons between measured $R_{\mathrm{g}}$. Interestingly, a mild compaction of G1-G3 from 3.4 to $3.2 \mathrm{~nm}$ was observed upon addition of $1 \mathrm{mM}$ calcium. This $R_{\mathrm{g}}$ of $3.2 \mathrm{~nm}$ fits well with the $R_{\mathrm{g}}$ of A1-A3 in the presence of $500 \mu \mathrm{M}$ calcium, which is dominated by active conformers. It is thus tempting to speculate that this slight calcium-induced compaction of G1-G3 may result from a shift between open (Int2) and active conformations through stabilization of the G2:G3 interface by calcium binding at the type II site of G2. In addition, the same study showed that mutations in the G2G3 linker could induce $\mathrm{Ca}^{2+}$-dependent severing activity in G1-G3. The mutants were found to adopt a collapsed state in the absence of calcium $\left(R_{\mathrm{g}}=2.6 \mathrm{~nm}\right.$ similar to inactive $\left.\mathrm{A} 1-\mathrm{A} 3\right)$ and expand in the presence of $1 \mathrm{mM}$ calcium to $R_{\mathrm{g}}=3.8 \mathrm{~nm}$. These dimensions are too large to represent exclusively active conformers and suggest the presence of fully expanded conformers, possibly resulting from destabilization of the linker structure. These results, together with the predominance of the open state of $\mathrm{A} 1-\mathrm{A} 3$ at high calcium concentrations, the changes in dynamics associated with the binding of calcium ions to the A2A 3 linker observed in MDS, and sequence divergence in this region between adseverin and gelsolin indicate that the linker between domains 2 and 3 plays a critical role in controlling protein dynamics by tuning the relative stability of the inactive, intermediate and active conformations.

In conclusion, we have drawn a quantitative picture of the conformational landscape of $\mathrm{A} 1-\mathrm{A} 3$ and provided structural insights into how calcium binding induces large conformational changes by affecting the relative stability of the accessible conformational states of A1-A3. Our biophysical characterization of A1-A3 in solution helps in explaining the apparent structural similarity yet different calcium-regulated dynamics that characterize the N-terminal halves of adseverin and gelsolin. The origin of these differences is best understood in the context of the full-length proteins. In the absence of a gelsolin C-terminal $\alpha$-helix latch, adseverin requires tighter interactions than gelsolin in the A1/A3 latch to maintain calcium control of activation.

\section{Methods}

Cloning, expression and purification. The cloning and Escherichia coli expression of the N-terminal halves of adseverin (A1-A3, residues 1-365) and gelsolin (G1-G3, residues 25-372) have been described previously in refs 23,20. Briefly, the constructs were engineered into a modified pET-21d $(+)$ (Novagen) plasmid, pSY5, to encode an 8-histidine tag, followed by a PreScission protease cleavage site, 
ahead of the $\mathrm{N}$ terminus of the proteins. The A1-A3 M310D and E314S mutants were generated using the QuikChange site-directed mutagenesis kit (Stratagene) and the following oligonucleotide pairs: $5^{\prime}$-CCCAAGAGAGGAAGGCTGCAG ATAAGACAGCTGAAGAATTTC- ${ }^{\prime} ; 5^{\prime}$-GAAATTCTTCAGCTGTCTTATCT GCAGCCTTCCTCTCTTGGG-3' for M310D and $5^{\prime}$-GGCTGCAATGAAGACA GCTTCAGAATTTCTACAGCAAATG-3'; 5'CATTTGCTGTAGAAATTCTGAA GCTGTCTTCATTGCAGCC- $3^{\prime}$ for E314S. Constructs were transformed into E. coli Rosetta 2 (DE3) cells (Novagen) for expression according to the manufacturer's protocol. Overnight starter cultures were prepared by growing the cells in LB liquid media supplemented with $100 \mu \mathrm{g} \mathrm{ml}^{-1}$ ampicillin and $34 \mu \mathrm{g} \mathrm{ml}^{-1}$ chloramphenicol at $37^{\circ} \mathrm{C}$ with shaking at 250 r.p.m. for $16 \mathrm{~h}$. Fresh LB liquid media for expression of each construct was inoculated with $50 \mathrm{ml}$ of overnight starter culture per liter, and incubated with shaking at 250 r.p.m. at $37^{\circ} \mathrm{C}$ until cell density reached $\mathrm{OD}_{600}=0.6$. Proteins were expressed by induction with $1 \mathrm{mM}$ isopropyl $\beta$-D-1-thiogalactopyranoside at $23^{\circ} \mathrm{C}$ overnight. Cells were harvested by centrifugation at $3,700 \mathrm{~g}$ for $30 \mathrm{~min}$. The pellets were subsequently resuspended in $100 \mathrm{ml}$ binding buffer $(50 \mathrm{mM}$ Tris- $\mathrm{HCl}(\mathrm{pH} 8.0), 500 \mathrm{mM}, \mathrm{NaCl}$ and $20 \mathrm{mM}$ imidazole), and disrupted by $2 \mathrm{mg} \mathrm{ml}^{-1}$ lysozyme treatment for $1 \mathrm{~h}$ at $4{ }^{\circ} \mathrm{C}$ followed by sonication.The suspensions were clarified by centrifugation at $32,000 \mathrm{~g}$ for $45 \mathrm{~min}$ in an SS-34 rotor in a RC 5C Plus centrifuge (Sorvall) at $4{ }^{\circ} \mathrm{C}$. Nickelnitrilotriacetic acid (Ni-NTA) beads $(2 \mathrm{ml})$ were added to the supernatant and mixed continuously for $30 \mathrm{~min}$ at $4{ }^{\circ} \mathrm{C}$. The beads were pelleted at $1,000 \mathrm{~g}$ at $4{ }^{\circ} \mathrm{C}$ for $15 \mathrm{~min}$, and resuspended in buffer containing $300 \mathrm{mM} \mathrm{NaCl}, 20 \mathrm{mM}$ imidazole, $10 \mathrm{mM}$ Tris- $\mathrm{HCl}(\mathrm{pH}$ 7.5). After washing the beads, proteins were eluted by raising the imidazole concentration to $250 \mathrm{mM}$. The His-tag was cleaved by addition of human rhinovirus $3 \mathrm{C}$ protease followed by overnight dialysis against $150 \mathrm{mM} \mathrm{NaCl}$ and $20 \mathrm{mM}$ Tris- $\mathrm{HCl}(\mathrm{pH} 7.5)$. Proteins were further purified by size-exclusion chromatography on a HiLoad 16/60 Superdex 200 prep grade column (GE Healthcare), first equilibrated with $125 \mathrm{ml}$ of $100 \mathrm{mM}$ EGTA, $20 \mathrm{mM}$ Tris-HCl (pH 7.2), $150 \mathrm{mM} \mathrm{NaCl}$, re-equilibrated with $250 \mathrm{ml}$ gel-filtration buffer $(0.2 \mathrm{mM}$ EGTA, $20 \mathrm{mM}$ Tris- $\mathrm{HCl}(\mathrm{pH} 7.2)$ and $150 \mathrm{mM} \mathrm{NaCl})$. Proteins were concentrated to $10 \mathrm{mg} \mathrm{ml}^{-1}$ by Ultra-15, MWCO $10 \mathrm{kDa}$, Amicon Ultra centrifugal filter units.

Crystallization and data collection. Crystallization was carried out by vapour diffusion using a Cartesian Technologies pipetting system ${ }^{40}$. Solutions containing $10 \mathrm{mg} \mathrm{ml}^{-1}$ of A1-A3 in $50 \mathrm{mM} \mathrm{NaCl}$ and $10 \mathrm{mM}$ Tris- $\mathrm{HCl}(\mathrm{pH} 7.5)$ supplemented with $5 \mathrm{mM}$ of either EDTA or $\mathrm{CaCl}_{2}$ crystallized in $20 \%$ polyethylene glycol 3,350, $100 \mathrm{mM}$ Bis-Tris propane (pH 7.5) or 30\% polyethylene glycol 6,000, $100 \mathrm{mM}$ HEPES (pH 7.0), respectively, which led to the crystal structures of iAl-A3 and aA3. Crystals were frozen in liquid nitrogen after being soaked in a mother liquor solution supplemented with $25 \%$ glycerol. Diffraction data were recorded on the ID29 and ID14-2 beamlines at the European Synchrotron Radiation Facility, Grenoble, France. Wavelength was 1.033 and $0.933 \AA$ for iA1-A3 and aA3, respectively. All data were automatically processed by xia2 (ref. 41).

Structure determination and refinement. Calcium-free iA1-A3 and calciumbound aA3 structures were solved by molecular replacement using the calcium-free $\mathrm{N}$-terminal half of gelsolin, iG1-G3, excised from PDB 1D0N (ref. 26) or the calcium-bound gelsolin domain 3, aG3 excised from PDB 1RGI (ref. 18) as search models in PHASER ${ }^{42}$. The molecular replacement solutions were subjected to repetitive rounds of restrained refinement in PHENIX ${ }^{43}$ and Autobuster ${ }^{44}$, and manual building in $\mathrm{COOT}^{45}$. Translation/Libration/Screw (TLS) parameters were included in the final round of refinement. The CCP4 programme suite ${ }^{46}$ was used for coordinate manipulations. The structures were validated with Molprobity ${ }^{47} .97$ and $99 \%$ of residues were in the Ramachandran-favoured region for iA1-A3 and aA3, respectively. Refinement statistics are given in Table 1 and a portion of the electron density map for each structure is shown in Supplementary Fig. 9.

SAXS experiments. Protein samples $\left(10 \mathrm{mg} \mathrm{ml}^{-1}\right.$ in $0.2 \mathrm{mM}$ EGTA, $20 \mathrm{mM}$ Tris- $\mathrm{HCl}(\mathrm{pH} 7.2)$ and $150 \mathrm{mM} \mathrm{NaCl}$ ) were mixed with $10 \times$ calcium or EGTA stock solutions to achieve the required free calcium concentrations, which were calculated using the Ca-EGTA Calculator v1.3 webserver (http://maxchelator.stanford.edu/CaEGTA-TS.htm) ${ }^{48}$. SAXS data were collected at the European Synchrotron Radiation Facility on beamline ID14-3. The sample-to-detector distance was $1 \mathrm{~m}$ and the wavelength of the X-rays was $0.0995 \mathrm{~nm}$. Data acquisition was performed at $20^{\circ} \mathrm{C}$. Protein concentrations ranged from 1 to $8 \mathrm{mg} \mathrm{ml}^{-1}$. Data reduction was performed using the established procedure available at ID14-3, and buffer background runs were subtracted from sample runs. The radius of gyration was determined with the programme PRIMUS ${ }^{49}$ according to the Guinier approximation at low $Q$ values, in a $Q . R_{g}$ range up to 1.3 :

$$
\ln I(Q)=\ln I(0)-\frac{R_{g}^{2} Q^{2}}{3}
$$

The pairwise distance distribution functions, $P(r)$, were calculated with the programme $\mathrm{GNOM}^{50}$. Molecular weights were estimated based on ref. 51 .

Ensemble generation. An ensemble approach was used to model the N-terminal half of adseverin (A1-A3). We employed all-atom coarse-grained $\mathrm{MDS}^{52}$ to generate candidate structures based on available X-ray crystallographic data.
Starting coordinates for the inactive state (I) were obtained from the crystal structure of calcium-free $\mathrm{N}$-terminal half of adseverin or from a homology model based on PDB 1RGI for the active structure $(\mathrm{A})^{18}$. Complete models including the missing disordered termini were generated in Modeller ${ }^{53}$. An additional homology model based on the structure of the gain-of-function mutant of the actin capping protein CapG (PDB 2J72; ref. 54), which differs from the active structure by having a different interface between domains 2 and 3, was also generated and used as a qualitative control of the accuracy of the ensemble optimization approach. Starting conformers for intermediates 1 and 2 (Int 1 and Int2) were obtained by dissociating domain 3 and then domain 1 from the iA1-A3 model. This was performed by defining and altering backbone torsions within the interdomain linker regions in VEGA $Z^{55}$. An additional model for Int2 was generated by dissociating domain 3 from domain 2 in the active structure, leading to an alternative conformer of Int2 where the alpha helix in the A2A3 linker is folded. Models of A1-A3 (I, Int1, Int2, $\mathrm{A}$ and the CapG-derived model) were simulated in GROMACS ${ }^{56}$ using an atomistic coarse-grained structure-based model ${ }^{57,58}$. A timestep of 0.0005 time units was used and the simulation was coupled to a temperature bath via Langevin dynamics. 10,000 Snapshots were extracted from each simulation, and a 1,000 models from each simulation were randomly dispatched into 10 independent ensembles of 6,000 models.

Ensemble optimization. For each model from each ensemble, theoretical SAXS patterns were calculated with the programme CRYSOL $^{59}$, and ensemble optimization fitting was performed for each of the 10 independent ensembles with $\mathrm{GAJOE}^{60}$. GAJOE uses a genetic algorithm to select from a large pool of conformers optimized sub-ensembles that minimize the discrepancy between the experimental and calculated curves $\chi_{\exp }$ according to the following equation:

$$
\chi_{\exp }^{2}=\frac{1}{K-1} \sum_{j=1}^{K}\left[\frac{\mu I\left(Q_{j}\right)-I_{\exp }\left(Q_{j}\right)}{\sigma\left(Q_{j}\right)}\right]^{2}
$$

where $K$ is the number of points in the experimental curve, $\sigma$ is the s.d. and $\mu$ is a scaling factor. The ensemble size was varied from 1 to 50 models and variations in the quality of the fit to the experimental data $\chi_{\exp }$ were monitored in order to analyse protein flexibility (Supplementary Fig. 6). Additional validation of the ensemble calculations was also performed by fitting the experimental data to pool ensembles including only I, A or I + A models, which yielded fits of poorer quality than when using ensembles that included the intermediate states (I+Int $1+$ Int2 + A; Supplementary Table 1 and Supplementary Fig. 3). In order to build conformational landscapes, 50 models were selected per ensemble, resulting in $50 \times 10=500$ optimized models. Interdomain distances were extracted from the models using GROMACS routines and then used to calculate two-dimensional histograms (using a binning of $15 \times 15$ ). The relative populations of conformers were counted in each optimized ensemble and averaged for each experimental SAXS profile to gain insight into the mechanism of calcium activation.

Explicit solvent MDS. Classical MD simulations of iA1-A3 in the presence and absence of calcium were performed using GROMACS 4 (ref. 56) and the AMBER99SB-ILDN force field ${ }^{61}$. At the beginning of each simulation, the protein was immersed in a box of SPC/E water, with a minimum distance of $1.0 \mathrm{~nm}$ between protein atoms and the edges of the box. An amount of $50 \mathrm{mM}$ of either $\mathrm{CaCl}_{2}$ or $\mathrm{NaCl}$ were then added using genion. Long-range electrostatics were treated with the particle-mesh Ewald summation ${ }^{62}$. Bond lengths were constrained using the P-LINCS algorithm. The integration timestep was $5 \mathrm{fs}$. The v-rescale thermostat and the Parrinello-Rahman barostat were used to maintain a temperature of $300 \mathrm{~K}$ and a pressure of 1 a.t.m. Each system was energy minimized using 1,000 steps of steepest descent and equilibrated for 200 ps with restrained protein heavy atoms. For each system, two independent production simulations were obtained by using different initial velocities. The aggregated simulation time was 200 and 2,000 ns for the simulations in the absence and presence of calcium, respectively. r.m.s.d. and root mean square fluctuations were calculated using GROMACS routines.

Actin depolymerization assays. Polymerization buffer (final concentration, $50 \mathrm{mM} \mathrm{KCl}, 0.2 \mathrm{mM}$ ATP, $2 \mathrm{mM} \mathrm{MgCl}_{2}, 0.5 \mathrm{mM}$ dithiothreitol, $1 \mathrm{mM}$ EGTA and $50 \mathrm{mM}$ HEPES, pH 7.5) was added to $10 \%$ pyrene-labelled G-actin $(6 \mu \mathrm{M})$ in 96-well, flat-bottomed plates (Corning), and was incubated for $30 \mathrm{~min}$ to allow the formation of F-actin. Calcium was then added to obtain the required free calcium concentration. Reactions were equilibrated for $1 \mathrm{~h}$ before $6 \mu \mathrm{M}$ of the respective proteins (A1-A3, A1-A3-M310D, A1-A3-E314S or G1-G3) were added. The final volume in each well was $100 \mu \mathrm{l}$. Fluorescence intensity was measured kinetically at excitation and emission wavelengths of 365 and $407 \mathrm{~nm}$, respectively, using a Safire2 fluorimeter (Tecan).

\section{References}

1. Silacci, P. et al. Gelsolin superfamily proteins: key regulators of cellular functions. Cell. Mol. Life Sci. 61, 2614-2623 (2004). 
2. Ghoshdastider, U., Popp, D., Burtnick, L. D. \& Robinson, R. C. The expanding superfamily of gelsolin homology domain proteins. Cytoskeleton (Hoboken) 70, 775-795 (2013).

3. Nag, S., Larsson, M. r., Robinson, R. C. \& Burtnick, L. D. Gelsolin: the tail of a molecular gymnast. Cytoskeleton 70, 360-384 (2013).

4. Rodriguez Del Castillo, A. et al. Chromaffin cell scinderin, a novel calcium-dependent actin filament-severing protein. EMBO J. 9, 43-52 (1990).

5. Vitale, M. L., Rodriguez Del Castillo, A., Tchakarov, L. \& Trifaro, J. M. Cortical filamentous actin disassembly and scinderin redistribution during chromaffin cell stimulation precede exocytosis, a phenomenon not exhibited by gelsolin. J. Cell Biol. 113, 1057-1067 (1991).

6. Dumitrescu Pene, T., Rose, S. D., Lejen, T., Marcu, M. G. \& Trifaro, J. M. Expression of various scinderin domains in chromaffin cells indicates that this protein acts as a molecular switch in the control of actin filament dynamics and exocytosis. J. Neurochem. 92, 780-789 (2005).

7. Hassanpour, S., Jiang, H., Wang, Y., Kuiper, J. W. \& Glogauer, M. The actin binding protein adseverin regulates osteoclastogenesis. PLOS ONE 9, e109078 (2014).

8. Zunino, R. et al. Expression of scinderin in megakaryoblastic leukemia cells induces differentiation, maturation, and apoptosis with release of plateletlike particles and inhibits proliferation and tumorigenesis. Blood 98, 2210-2219 (2001).

9. Nurminsky, D., Magee, C., Faverman, L. \& Nurminskaya, M. Regulation of chondrocyte differentiation by actin-severing protein adseverin. Dev. Biol. 302, 427-437 (2007).

10. Li, X. et al. Expression and function of the actin-severing protein adseverin in the proliferation, migration, and differentiation of dental pulp cells. J Endod. 41, 493-500 (2015)

11. Miura, N. et al. Adseverin: a novel cisplatin-resistant marker in the human bladder cancer cell line HT1376 identified by quantitative proteomic analysis. Mol. Oncol. 6, 311-322 (2012).

12. Liu, H., Shi, D., Liu, T., Yu, Z. \& Zhou, C. Lentivirus-mediated silencing of SCIN inhibits proliferation of human lung carcinoma cells. Gene 554, 32-39 (2014).

13. Chen, X. M. et al. Suppression of scinderin modulates epithelialmesenchymal transition markers in highly metastatic gastric cancer cell line SGC7901. Mol. Med. Rep. 10, 2327-2333 (2014).

14. Wang, D. et al. Suppression of SCIN inhibits human prostate cancer cell proliferation and induces G0/G1 phase arrest. Int. J. Oncol. 44, 161-166 (2013).

15. Lin, K. M., Mejillano, M. \& Yin, H. L. Ca2 + regulation of gelsolin by its C-terminal tail. J. Biol. Chem. 275, 27746-27752 (2000).

16. Ditsch, A. \& Wegner, A. Two low-affinity $\mathrm{Ca}(2+)$-binding sites of gelsolin that regulate association with actin. Eur. J. Biochem. 229, 512-516 (1995).

17. Robinson, R. C. et al. Domain movement in gelsolin: a calcium-activated switch. Science 286, 1939-1942 (1999).

18. Burtnick, L. D., Urosev, D., Irobi, E., Narayan, K. \& Robinson, R. C. Structure of the N-terminal half of gelsolin bound to actin: roles in severing, apoptosis and FAF. EMBO J. 23, 2713-2722 (2004).

19. Chumnarnsilpa, S. et al. Calcium ion exchange in crystalline gelsolin. J. Mol. Biol. 357, 773-782 (2006).

20. Nag, S. et al. $\mathrm{Ca} 2+$ binding by domain 2 plays a critical role in the activation and stabilization of gelsolin. Proc. Natl Acad. Sci. USA 106, 13713-13718 (2009).

21. Lueck, A., Yin, H. L., Kwiatkowski, D. J. \& Allen, P. G. Calcium regulation of gelsolin and adseverin: a natural test of the helix latch hypothesis. Biochemistry 39, 5274-5279 (2000).

22. Choe, H. et al. The calcium activation of gelsolin: insights from the $3 \mathrm{~A}$ structure of the G4-G6/actin complex. J. Mol. Biol. 324, 691-702 (2002).

23. Chumnarnsilpa, S. et al. The crystal structure of the C-terminus of adseverin reveals the actin-binding interface. Proc. Natl Acad. Sci. USA 106, 13719-13724 (2009).

24. Maekawa, S. \& Sakai, H. Inhibition of actin regulatory activity of the $74-\mathrm{kDa}$ protein from bovine adrenal medulla (adseverin) by some phospholipids. J. Biol. Chem. 265, 10940-10942 (1990).

25. Janmey, P. A. \& Stossel, T. P. Modulation of gelsolin function by phosphatidylinositol 4,5-bisphosphate. Nature 325, 362-364 (1987).

26. Burtnick, L. D. et al. The crystal structure of plasma gelsolin: implications for actin severing, capping, and nucleation. Cell 90, 661-670 (1997).

27. Wang, H. et al. Helix straightening as an activation mechanism in the gelsolin superfamily of actin regulatory proteins. J. Biol. Chem. 284, 21265-21269 (2009).

28. Ashish et al. Global structure changes associated with $\mathrm{Ca} 2+$ activation of full-length human plasma gelsolin. J. Biol. Chem. 282, 25884-25892 (2007).

29. Sakurai, T., Kurokawa, H. \& Nonomura, Y. The Ca2(+)-dependent actin filament-severing activity of $74-\mathrm{kDa}$ protein (adseverin) resides in its NH2-terminal half. J. Biol. Chem. 266, 4581-4585 (1991).

30. Chaponnier, C., Janmey, P. A. \& Yin, H. L. The actin filament-severing domain of plasma gelsolin. J. Cell Biol. 103, 1473-1481 (1986).
31. Peddada, N. et al. Global shapes of F-actin depolymerization-competent minimal gelsolins: insight into the role of $\mathrm{g} 2$-g3 linker in $\mathrm{pH} / \mathrm{Ca} 2+$ insensitivity of the first half. J. Biol. Chem. 288, 28266-28282 (2013).

32. Bernado, P. Effect of interdomain dynamics on the structure determination of modular proteins by small-angle scattering. Eur. Biophys. J. 39, 769-780 (2009).

33. Leyrat, C., Renner, M., Harlos, K., Huiskonen, J. T. \& Grimes, J. M. Structure and self-assembly of the calcium binding matrix protein of human metapneumovirus. Structure 22, 136-148 (2014).

34. Garcia, A. G., Garcia-De-Diego, A. M., Gandia, L., Borges, R. \& Garcia-Sancho, J. Calcium signaling and exocytosis in adrenal chromaffin cells. Physiol. Rev. 86, 1093-1131 (2006).

35. Huang, Y. \& Liu, Z. Kinetic advantage of intrinsically disordered proteins in coupled folding-binding process: a critical assessment of the "fly-casting" mechanism. J. Mol. Biol. 393, 1143-1159 (2009).

36. Shoemaker, B. A., Portman, J. J. \& Wolynes, P. G. Speeding molecular recognition by using the folding funnel: the fly-casting mechanism. Proc. Natl Acad. Sci. USA 97, 8868-8873 (2000).

37. Tchakarov, L., Vitale, M. L., Jeyapragasan, M., Rodriguez Del Castillo, A. \& Trifaro, J. M. Expression of scinderin, an actin filament-severing protein, in different tissues. FEBS Lett. 268, 209-212 (1990).

38. Ma, B., Tsai, C. J., Haliloglu, T. \& Nussinov, R. Dynamic allostery: linkers are not merely flexible. Structure 19, 907-917 (2011).

39. Krissinel, E. \& Henrick, K. Inference of macromolecular assemblies from crystalline state. J. Mol. Biol. 372, 774-797 (2007).

40. Walter, T. S. et al. A procedure for setting up high-throughput nanolitre crystallization experiments. Crystallization workflow for initial screening, automated storage, imaging and optimization. Acta Crystallogr. D Biol. Crystallogr. 61, 651-657 (2005).

41. Winter, G., Lobley, C. M. \& Prince, S. M. Decision making in xia2. Acta Crystallogr. D Biol. Crystallogr. 69, 1260-1273 (2013).

42. McCoy, A. J. et al. Phaser crystallographic software. J. Appl. Crystallogr. 40, 658-674 (2007).

43. Adams, P. D. et al. PHENIX: a comprehensive Python-based system for macromolecular structure solution. Acta Crystallogr. D Biol. Crystallogr. 66, 213-221 (2010).

44. Blanc, E. et al. Refinement of severely incomplete structures with maximum likelihood in BUSTER-TNT. Acta Crystallogr. D Biol. Crystallogr. 60, 2210-2221 (2004).

45. Emsley, P., Lohkamp, B., Scott, W. G. \& Cowtan, K. Features and development of Coot. Acta Crystallogr. D Biol. Crystallogr. 66, 486-501 (2010).

46. Winn, M. D. et al. Overview of the CCP4 suite and current developments. Acta Crystallogr. D Biol. Crystallogr. 67, 235-242 (2011).

47. Chen, V. B. et al. MolProbity: all-atom structure validation for macromolecular crystallography. Acta Crystallogr. D Biol. Crystallogr. 66, 12-21 (2010).

48. Schoenmakers, T. J., Visser, G. J., Flik, G. \& Theuvenet, A. P. CHELATOR: an improved method for computing metal ion concentrations in physiological solutions. Biotechniques 12, 870-874 876-9 (1992).

49. Svergun, D. I., Konarev, P. V., Volkov, V. V., Sokolova, A. V. \& Koch, M. H. J. PRIMUS: a Windows PC-based system for small-angle scattering data analysis. J. Appl. Crystallogr. 36, 1277-1282 (2003).

50. Semenyuk, A. V. \& Svergun, D. I. Gnom - a Program package for small-angle scattering data-processing. J. Appl. Crystallogr. 24, 537-540 (1991).

51. Rambo, R. P. \& Tainer, J. A. Accurate assessment of mass, models and resolution by small-angle scattering. Nature 496, 477-481 (2013).

52. Onuchic, J. N. et al. An all-atom structure-based potential for proteins: Bridging minimal models with all-atom empirical forcefields. Proteins 75, 430-441 (2009).

53. Eswar, N., Eramian, D., Webb, B., Shen, M. Y. \& Sali, A. Protein structure modeling with MODELLER. Methods Mol. Biol. 426, 145-159 (2008).

54. Zhang, Y. et al. A CapG gain-of-function mutant reveals critical structural and functional determinants for actin filament severing. EMBO J. 25, 4458-4467 (2006).

55. Vistoli, G., Pedretti, A. \& Villa, L. VEGA - An open platform to develop chemo-bio-informatics applications, using plug-in architecture and script programming. Journal of Computer-Aided Molecular Design 18, 167-173 (2004).

56. Hess, B., Kutzner, C., van der Spoel, D. \& Lindahl, E. GROMACS 4: algorithms for highly efficient, load-balanced, and scalable molecular simulation. J. Chem. Theory Comput. 4, 435-447 (2008).

57. Noel, J. K., Whitford, P. C., Sanbonmatsu, K. Y. \& Onuchic, J. N. SMOG@ctbp: simplified deployment of structure-based models in GROMACS. Nucleic Acids Res. 38, W657-W661 (2010).

58. Whitford, P. C. et al. An all-atom structure-based potential for proteins: bridging minimal models with all-atom empirical forcefields. Proteins 75, 430-441 (2009). 
59. Svergun, D., Barberato, C. \& Koch, M. H. J. CRYSOL-a program to evaluate $\mathrm{X}$-ray solution scattering of biological macromolecules from atomic coordinates. J. Appl. Crystallogr. 28, 768-773 (1995).

60. Bernado, P., Mylonas, E., Petoukhov, M. V., Blackledge, M. \& Svergun, D. I. Structural characterization of flexible proteins using small-angle X-ray scattering. J. Am. Chem. Soc. 129, 5656-5664 (2007).

61. Lindorff-Larsen, K. et al. Improved side-chain torsion potentials for the Amber ff99SB protein force field. Proteins 78, 1950-1958 (2010).

62. Essmann, U. et al. A smooth particle mesh Ewald method. J. Chem. Phys. 103, 8577-8593 (1995).

\section{Acknowledgements}

We thank the European Synchrotron Radiation Facility (Grenoble, France) for beamtime, and the staff of beamlines ID29, ID14-2 and ID14-3 for assistance with crystallography and SAXS data collection.

\section{Author contributions}

S.C., R.C.R., J.M.G. and C.L. designed the research; S.C., J.M.G. and C.L. performed experiments; S.C., R.C.R., J.M.G. and C.L. analysed data; and S.C., R.C.R., J.M.G. and C.L. wrote the paper.

\section{Additional information}

Accession codes. The final refined coordinates and structure factors have been deposited in the PDB with accession codes $5 \mathrm{~A} 1 \mathrm{~K}$ and $5 \mathrm{~A} 1 \mathrm{M}$.

Supplementary Information accompanies this paper at http://www.nature.com/ naturecommunications

Competing financial interests: The authors declare no competing financial interests.

Reprints and permission information is available online at http://npg.nature.com/ reprintsandpermissions/

How to cite this article: Chumnarnsilpa, S. et al. Calcium-controlled conformational choreography in the $\mathrm{N}$-terminal half of adseverin. Nat. Commun. 6:8254 doi: $10.1038 /$ ncomms9254 (2015).

(c) (i) This work is licensed under a Creative Commons Attribution 4.0 International License. The images or other third party material in this article are included in the article's Creative Commons license, unless indicated otherwise in the credit line; if the material is not included under the Creative Commons license, users will need to obtain permission from the license holder to reproduce the material. To view a copy of this license, visit http://creativecommons.org/licenses/by/4.0/ 